\title{
Multiuser Detection for Nonlinear MIMO Uplink
}

\author{
Sheng Chen, Fellow, IEEE, Soon Xin Ng, Senior Member, IEEE, Emad Khalaf, Ali Morfeq, Naif Alotaibi
}

\begin{abstract}
For the multiple-input multiple-output (MIMO) uplink employing high-order quadrature amplitude modulation (QAM) signaling and with nonlinear high power amplifiers (HPAs) at mobile users' transmitters, the existing multiuser detection methods can no longer be applied. We propose a novel nonlinear multiuser detection scheme for the nonlinear MIMO uplink. Specifically, we adopt an effective B-spline parameterization of the nonlinear transmit HPAs and derive an efficient and accurate algorithm to identify the nonlinear MIMO uplink channel, including the nonlinear B-spline model of the nonlinear transmit HPAs and the estimate of the linear MIMO channel matrix. Moreover, as the direct result of this nonlinear MIMO channel identification, the B-spline inverse model of nonlinear transmit HPAs can readily be identified. The nonlinear multiuser detection can be effectively implemented by the zeroforcing linear detection based on the estimated linear MIMO channel and followed by compensating the nonlinear distortion of the nonlinear transmit HPAs based on the estimated B-spline inverse model. An extensive simulation investigation is performed to demonstrate the effectiveness of our proposed nonlinear multiuser detection scheme for nonlinear MIMO uplink with high-order QAM signaling.
\end{abstract}

Index Terms-Multi-input multi-output (MIMO), uplink, multiuser detection, nonlinear high power amplifier, nonlinear MIMO channel, complex-valued B-spline neural network

\section{INTRODUCTION}

In recent years, multiple-input multiple-output (MIMO) systems have attracted considerable attention from both academia and industry, owing to their capability of significantly increasing the reliability and/or bandwidth efficiency of communication systems [1]-[10]. In particular, the MIMO technology offers a practical and efficiency means of space division multiple access (SDMA) for supporting multiple users with the same resource block, and hence it dramatically increases the cellular network user capacity. Most of the existing MIMO system designs assume the linear MIMO channel [11]-[25], and this assumption is valid when the high power amplifier (HPA) at transmitter operates within its linear dynamic range. In the current and future wireless systems, high-order quadrature amplitude modulation (QAM) signaling [26] is increasingly adopted to support high-throughput applications. The highorder QAM signaling achieves high bandwidth efficiency but leads to high peak-to-average power ratio (PAPR) of the resulting transmit signal. Since practical HPAs exhibit nonlinear saturation characteristics [27]-[31], the high PAPR signal may

S. Chen and S. X. Ng are with School of Electronics and Computer Science, University of Southampton, Southampton SO17 1BJ, UK (E-mails: sqc@ecs.soton.ac.uk, sxn@ecs.soton.ac.uk). S. Chen is also with King Abdulaziz University, Jeddah 21589, Saudi Arabia.

E. Khalaf, A. Morfeq and N. Alotaibi are with Electrical and Computer Engineering Department, Faculty of Engineering, King Abdulaziz University, Jeddah 21589, Saudi Arabia (E-mails: ekhalaf@kau.edu.sa, morfeq@kau.edu.sa,ndalotabi@kau.edu.sa).

This project was funded by the Deanship of Scientific Research (DSR) at King Abdulaziz University, Jeddah, under grant no.RG-3-135-40. The authors, therefore, acknowledge with thanks DSR technical and financial support. drive the HPA at transmitter into the nonlinear saturation region, and consequently the assumption of the linear MIMO channel model no longer holds.

A classical way of avoiding the nonlinearity of the transmitter HPA is to apply output back-off (OBO). By lowering the average transmit power sufficiently away from the saturation power level of the HPA, hopefully the peak transmitted signal may still fall in the near-linear operating region of the HPA. For high-order QAM signals, however, OBO must be very severe to be effective. But such a large OBO will dramatically reduce the efficiency of the HPA, and more importantly it may not meet the required link power budget, especially for the mobile users (MUs) at cell edge. For downlink, an effective approach to compensate for the nonlinear distortions of HPA is to implement a digital predistorter at the base station (BS) transmitter, and various predistorter techniques have been developed [32]-[38]. Implementing the predistorter is very practical and attractive for the downlink, because the BS has the sufficient hardware and software capacities to accommodate the hardware and computational requirements for implementing digital predistorter. In uplink, however, the predistorter option is not viable because it is extremely difficult for a pocket-size handset to absorb the required hardware and computational complexity. Consequently, the BS receiver must deal with the nonlinear distortions of the transmitter HPA.

With the nonlinear HPAs at MUs' transmitters, the uplink channel is a nonlinear MIMO Hammerstein system with the received signals further impaired by the channel additive white Gaussian noise (AWGN). Nonlinear multiuser detection is extremely challenging, and very few works have tackled this difficult task. In the studies [39]-[41], the MIMO Volterra model is employed to identify the MIMO Hammerstein communication channel. The Volterra model is cumbersome as it contains a very large number of model parameters. Hence it requires a huge number of training pilots and imposes heavy computational burden. Furthermore, the estimation algorithm of [39]-[41] requires a very large number of iterations to converge. Even after acquiring the MIMO Volterra channel model, the real task of multiuser detection remains to be resolved. In [39], it is suggested that the maximum likelihood (ML) detection is applied. However, with such a large MIMO Volterra channel model and the high-order QAM signaling, the optimal ML detector is computationally prohibitive. No practical low-complexity nonlinear multiuser detection is provided in [39]-[41] for high-order QAM signaling. More strangely, the studies [39]-[41] also consider the phase shift keying (PSK) signaling. The PAPR of PSK is unity. Therefore, the practical HPAs [27]-[31] do not cause amplitude distortion and the phase shift of the HPA's output is constant for all the PSK symbols. Effectively, the MIMO channel is always linear for PSK. To the best knowledge of the authors, no work to date has addressed the problem of multiuser detection for nonlinear 
MIMO uplink with high-order QAM signaling.

Against the above background, in this paper, we develop a new and effective approach for multiuser detection of the nonlinear MIMO uplink with high-order QAM signaling. Our novel contributions are summarized as follows.

- We adopt the B-spline neural networks [42]-[45] to provide an optimal and efficient model for the MUs' HPAs, which yields an effective parameterization of the nonlinear block of the MIMO Hammerstein channel, as well as to provide the inversion model of the MUs' HPAs, which is essential in multiuser detection.

- We develop a highly efficient and accurate estimation algorithm to identify the MIMO Hammerstein channel, including the nonlinear B-spline model of the MUs' HPAs and the estimate of the linear MIMO channel matrix. Moreover, as the consequence of this MIMO Hammerstein channel identification, the B-spline inverse model of the transmitters' nonlinear HPAs can readily be identified.

- With the estimates of the linear MIMO channel matrix and the inverse model of the HPAs, the multiuser detection can be carried out by the zero-forcing (ZF) detection based on the estimated linear MIMO channel matrix followed by the inversion of the HPAs to compensate the nonlinear distortion of the HPAs.

- An extensive simulation investigation demonstrates the effectiveness of our proposed nonlinear multiuser detection approach for nonlinear MIMO uplink with high-order QAM signaling.

Before we proceed to our method, we emphasize that the proposed nonlinear multiuser detection approach for nonlinear MIMO uplink with high-order QAM signaling is not a simple extension of our previous works of nonlinear equalization for single-input single-output (SISO) Hammerstein channels [46][50]. Parameterization of the MIMO Hammerstein channel is much more challenging than that for the SISO Hammerstein channel. Moreover, the efficient identification algorithm developed for the SISO Hammerstein channel [46]-[50] cannot be applied to the case of MIMO Hammerstein channels. The parameterization and identification derived in this paper for MIMO Hammerstein channels are entirely new. More specifically, we need to derive a unique nonlinear MIMO channel parameterization capable of resolving the ordering and scaling ambiguities between the linear MIMO channel matrix and the multiple nonlinear transmitters. Furthermore, the single-loop two-stage alternating least squares (ALS) algorithm of [46][50] cannot be applied to this nonlinear MIMO channel model, and we have to develop a new two-loop three-stage ALS (TL3S-ALS) algorithm for the identification task.

\section{Nonlinear Mimo System Model}

As illustrated in Fig. 1, we consider the standard singlecarrier MIMO uplink where the BS is equipped with the $L$ antennas to support $M$ single-antenna MUs using the same resource block. The data symbol of the $m$ th MU at sample $k$ is denoted by $x_{m}(k)$ which takes the value from the $U$-QAM constellation

$$
\mathbb{X}=\{d(2 l-\sqrt{U}-1)+\mathbf{j} d(2 q-\sqrt{U}-1), 1 \leq l, q \leq \sqrt{U}\},
$$

where $2 d$ is the minimum distance between symbol points. In the equivalent baseband discrete-time domain, the transmitted signal of the $m$ th $\mathrm{MU}$ is given by

$$
w_{m}(k)=\Psi\left(x_{m}(k)\right), 1 \leq m \leq M,
$$

where $\Psi(\cdot)$ represents the nonlinear HPA at an MU's transmitter. The solid state power amplifier [30], [31] is typically employed, and the nonlinearity $\Psi(\cdot)$ of this type of HPA is defined by the HPA's amplitude response $A(r)$ and phase response $\Upsilon(r)$, given respectively by

$$
\begin{aligned}
& A(r)=\frac{g_{a} r}{\left(1+\left(\frac{g_{a} r}{A_{\text {sat }}}\right)^{2 \beta_{a}}\right)^{\frac{1}{2 \beta_{a}}},} \\
& \Upsilon(r)=\frac{\alpha_{\phi} r^{q_{1}}}{1+\left(\frac{r}{\beta_{\phi}}\right)^{q_{2}}} \text { [degree], }
\end{aligned}
$$

where $r$ denotes the amplitude of the input to the HPA, $g_{a}$ is the small signal's gain, $\beta_{a}$ is the smoothness factor and $A_{\text {sat }}$ is the saturation level, while the parameters of the phase response, $\alpha_{\phi}, \beta_{\phi}, q_{1}$ and $q_{2}$, are adjusted to match the specific amplifier's characteristics [30], [31]. The operating status of the HPA is specified by the OBO, which is defined as the ratio of the maximum output power $P_{\max }$ of the HPA to the average output power $P_{\text {aop }}$ of the HPA output signal, given by

$$
\mathrm{OBO}=10 \cdot \log _{10} \frac{P_{\max }}{P_{\text {aop }}}[\mathrm{dB}] .
$$

The smaller OBO is, the more the HPA is operating into the nonlinear saturation region.

The received signal vector $\boldsymbol{y}(k)=\left[y_{1}(k) \cdots y_{L}(k)\right]^{\mathrm{T}} \in \mathbb{C}^{L}$ at the BS is given by the well-known equivalent baseband discrete-time MIMO model

$$
\begin{aligned}
\boldsymbol{y}(k) & =\left[\begin{array}{cccc}
h_{1,1} & h_{1,2} & \cdots & h_{1, M} \\
h_{2,1} & h_{2,2} & \cdots & h_{2, M} \\
\vdots & \vdots & \ddots & \vdots \\
h_{L, 1} & h_{L, 2} & \cdots & h_{L, M}
\end{array}\right] \boldsymbol{w}(k)+\boldsymbol{n}(k) \\
& =\boldsymbol{H} \boldsymbol{w}(k)+\boldsymbol{n}(k),
\end{aligned}
$$

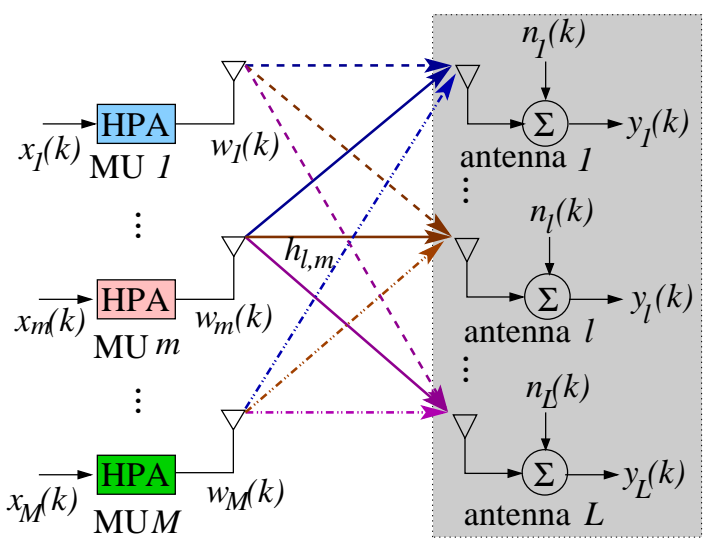

Fig. 1. MIMO uplink where BS employs $L$ antennas to support $M$ singleantenna mobile users with nonlinear transmit power amplifiers. 
where $\boldsymbol{w}(k)=\left[w_{1}(k) \cdots w_{M}(k)\right]^{\mathrm{T}} \in \mathbb{C}^{M}$ is the transmitted signal vector of the $M$ MUs, and $\boldsymbol{n}(k)=\left[n_{1}(k) \cdots n_{L}(k)\right]^{\mathrm{T}} \in$ $\mathbb{C}^{L}$ is the AWGN vector with the zero mean vector and the covariance matrix $E\left\{\boldsymbol{n}(k) \boldsymbol{n}^{\mathrm{H}}(k)\right\}=2 \sigma_{n}^{2} \boldsymbol{I}_{L}$, while the MIMO channel matrix $\boldsymbol{H} \in \mathbb{C}^{L \times M}$ whose $(l, m)$ th element $h_{l, m}$ denotes the coefficient of the channel linking the $m$ th MU to the $l$ th antenna of the BS. Clearly, parameterization of the nonlinear MIMO model (6) and (2) is not unique. Specifically, any proper scaling and ordering of the two components (6) and (2) will lead to the same MIMO output $\boldsymbol{y}(k)$. This will cause serious problems for the identification task.

In order to develop efficient identification algorithm, we need to derive a unique parameterization of the linear MIMO channel matrix and the $M$ nonlinear transmitters. Reexpress (6) equivalently as

$$
\begin{aligned}
\boldsymbol{y}(k)= & {\left[\begin{array}{cccc}
\frac{h_{1,1}}{h_{1,1}} & \frac{h_{1,2}}{h_{1,2}} & \cdots & \frac{h_{1, M}}{h_{1, M}} \\
\frac{h_{2,1}}{h_{1,1}} & \frac{h_{2,2}}{h_{1,2}} & \cdots & \frac{h_{2, M}}{h_{1, M}} \\
\vdots & \vdots & \ddots & \vdots \\
\frac{h_{L, 1}}{h_{1,1}} & \frac{h_{L, 2}}{h_{1,2}} & \cdots & \frac{h_{L, M}}{h_{1, M}}
\end{array}\right]\left[\begin{array}{c}
h_{1,1} \Psi\left(x_{1}(k)\right) \\
\frac{h_{1,2}}{h_{1,1}} h_{1,1} \Psi\left(x_{2}(k)\right) \\
\vdots \\
\frac{h_{1, M}}{h_{1,1}} h_{1,1} \Psi\left(x_{M}(k)\right)
\end{array}\right] } \\
& +\boldsymbol{n}(k) .
\end{aligned}
$$

According to (7), we can parametrise the linear MIMO channel matrix $\boldsymbol{H}$ in (6) equivalently as

$$
\boldsymbol{H}=\left[\begin{array}{cccc}
1 & 1 & \cdots & 1 \\
h_{2,1} & h_{2,2} & \cdots & h_{2, M} \\
\vdots & \vdots & \ddots & \vdots \\
h_{L, 1} & h_{L, 2} & \cdots & h_{L, M}
\end{array}\right]
$$

where for notational simplicity, we still denote this equivalent linear MIMO channel matrix as $\boldsymbol{H}$, and we can also express the nonlinear transmitters $\boldsymbol{w}(k)$ by

$$
w_{m}(k)=\zeta_{m} \Psi\left(x_{m}(k)\right), 1 \leq m \leq M,
$$

with $\zeta_{1}=1$, where $\zeta_{m} \in \mathbb{C}$ for $2 \leq m \leq M$.

Remark 1: The entries of the MIMO channel matrix $\boldsymbol{H}$ in (6) are generally independent random variables following the complex Gaussian distribution with zero mean and variance of 0.5 per dimension, i.e., $h_{l, m} \sim \mathcal{C N}(0,1), 1 \leq l \leq L$, $1 \leq m \leq M$. Since $\boldsymbol{H}$ is multiplicative with the model of the nonlinear HPAs, there are infinitely many pairs of the parameterization for $\boldsymbol{H}$ and the HPAs' model, and they are all equivalent. In fact, all the parameterizations $\boldsymbol{H} \boldsymbol{U} \boldsymbol{U}^{*} \boldsymbol{w}$ are equivalent, where $\boldsymbol{U} \in \mathbb{C}^{M \times M}$ is any unitary matrix. Moreover, for any particular given $\boldsymbol{U}^{*} \boldsymbol{w}$, we also have infinitely many pairs of the parameterization. We choose the particular parameterization for $\boldsymbol{H}$ given in (8) and for the HPAs' model given in (9). The 'equivalent' MIMO channel matrix (8) corresponds to divide the $m$ th column of the orignal MIMO channel matrix $\boldsymbol{H}$ in (6) by $h_{1, m}$ for $1 \leq m \leq M$, and the 'equivalent' HPAs' model (9) with $\zeta_{1}=1$ corresponds to absorb $h_{1,1}$ into the orignal HPA nonlinearity $\Psi(\cdot)$, which for notational convenience is still denoted as $\Psi(\cdot)$, with $\zeta_{m}=h_{1, m} / h_{1,1}$ for $2 \leq m \leq M$. This parameterization is essential for the identification algorithm to attain an efficient and unique estimate of the true MIMO Hammerstein channel.
If the MIMO channel matrix $\boldsymbol{H}$ is available at the BS, the ZF detection of $\boldsymbol{w}(k)$ is given by

$$
\widehat{\boldsymbol{w}}(k)=\left(\boldsymbol{H}^{\mathrm{H}} \boldsymbol{H}\right)^{-1} \boldsymbol{H}^{\mathrm{H}} \boldsymbol{y}(k),
$$

where $\widehat{\boldsymbol{w}}(k)=\left[\widehat{w}_{1}(k) \widehat{w}_{2}(k) \cdots \widehat{w}_{M}(k)\right]^{\mathrm{T}}$. Furthermore, if the inversions $\left(\zeta_{m} \Psi\right)^{-1}(\cdot)$ for $1 \leq m \leq M$ are known to the BS, the data detection is readily achieved with

$$
\widehat{x}_{m}(k)=\left(\zeta_{m} \Psi\right)^{-1}\left(\widehat{w}_{m}(k)\right), 1 \leq m \leq M .
$$

However, both $\boldsymbol{H}$ and $\left(\zeta_{m} \Psi\right)^{-1}(\cdot)$ for $1 \leq m \leq M$ are unknown to the BS. The main contribution of this paper is for the first time to develop a practical and efficient means of implementing this multiuser detection for nonlinear MIMO uplink with high-order QAM signaling.

\section{The Proposed Multiuser Detection Scheme}

As shown previously, multiuser detection for nonlinear MIMO uplink requires the knowledge of the MIMO channel matrix $\boldsymbol{H}$ as well as the inverse mappings of all the MUs' nonlinear HPAs, which are unknown to the BS. Note that during the training, the BS only has the training data $\{\boldsymbol{y}(k), \boldsymbol{x}(k)\}_{k=1}^{K}$. But the BS does not have $\boldsymbol{w}(k)$ and it cannot use the standard least squares (LS) method to estimate $\boldsymbol{H}$. We will extend the B-spline neural network approach of [45]-[50] to develop a new method of estimating $\boldsymbol{H}$ as well as $\zeta_{m} \Psi(\cdot)$ and $\left(\zeta_{m} \Psi\right)^{-1}(\cdot), 1 \leq m \leq M$.

\section{A. B-spline neural network parameterization}

We use a complex-valued B-spline neural network to model the nonlinear HPA (9). According to the physics of real-life HPA, the nonlinearity $\Psi(\cdot)$ satisfies the following conditions.

1) $\Psi(\cdot)$ is a one to one mapping, i.e., it is an invertible and continuous function.

2) $x_{R}$ and $x_{I}$ are upper and lower bounded by some finite and known real values, where $x=x_{R}+\mathrm{j} x_{I}$ denotes the input to the HPA $\Psi(\cdot)$. Furthermore, the distributions of $x_{R}$ and $x_{I}$ are identical.

Based on property 2), we have $U_{\min }<x_{\mathrm{s}}<U_{\max }$, where $U_{\min }$ and $U_{\max }$ are known finite real values, while $x_{\mathrm{s}} \in \mathbb{R}$ represents either $x_{R}$ or $x_{I}$.

To model a nonlinearity in the univariate dimension of $x_{\mathrm{s}}$, we use a univariate B-spline model with piecewise polynomial degree of $P_{o}$ and $N_{\mathrm{s}}$ basis functions. This univariate B-spline neural network is parametrized by the knot sequence specified by $\left(N_{\mathrm{s}}+P_{o}+1\right)$ knot values, $\left\{U_{0}, U_{1}, \cdots, U_{N_{\mathrm{s}}+P_{o}}\right\}$, with

$$
\begin{gathered}
U_{0}<U_{1}<\cdots<U_{P_{o}-2}<U_{P_{o}-1}=U_{\min }<U_{P_{o}}<\cdots< \\
U_{N_{\mathrm{s}}}<U_{N_{\mathrm{s}}+1}=U_{\max }<U_{N_{\mathrm{s}}+2}<\cdots<U_{N_{\mathrm{s}}+P_{o}} .
\end{gathered}
$$

At each end, there are $P_{o}-1$ external knots that are outside the input region $\left[U_{\min }, U_{\max }\right]$ and one boundary knot. As a result, the number of internal knots is given by $N_{\mathrm{s}}+1-P_{o}$. Given the set of predetermined knots (12), the set of $N_{\mathrm{s}}$ Bspline basis functions are formed using the De Boor recursion [42], yielding for $1 \leq l \leq N_{\mathrm{s}}+P_{o}$,

$$
B_{l}^{(\mathrm{s}, 0)}\left(x_{\mathrm{s}}\right)= \begin{cases}1, & \text { if } U_{l-1} \leq x_{\mathrm{s}}<U_{l}, \\ 0, & \text { otherwise }\end{cases}
$$


as well as for $l=1, \cdots, N_{\mathrm{s}}+P_{o}-p$ and $p=1, \cdots, P_{o}$,

$$
\begin{aligned}
B_{l}^{(\mathrm{s}, p)}\left(x_{\mathrm{s}}\right)= & \frac{x_{\mathrm{s}}-U_{l-1}}{U_{p+l-1}-U_{l-1}} B_{l}^{(\mathrm{s}, p-1)}\left(x_{\mathrm{s}}\right) \\
& +\frac{U_{p+l}-x_{\mathrm{s}}}{U_{p+l}-U_{l}} B_{l+1}^{(\mathrm{s}, p-1)}\left(x_{\mathrm{s}}\right) .
\end{aligned}
$$

The polynomial degree $P_{o}=3$ or 4 is often sufficient for modeling the nonlinearities encountered in most practical applications. The number of B-spline basis functions $N_{\mathrm{S}}$ should be sufficiently large to provide accurate approximation capability but not too large as to cause overfitting and to impose unnecessary computational complexity. Specifically, $N_{\mathrm{s}}=6$ to 10 is usually sufficient for accurately modeling in the finite and known interval $\left[U_{\min }, U_{\max }\right]$. The two boundary knots are obviously related to the known values $U_{\min }$ and $U_{\max }$, respectively. The $N_{\mathrm{s}}+1-P_{o}$ internal knots may be uniformly spaced in the interval $\left[U_{\min }, U_{\max }\right]$. Note that there exist no data for $x_{\mathrm{s}}<U_{\min }$ and $x_{\mathrm{s}}>U_{\max }$ in identification but it is desired that the B-spline model has certain extrapolating capability outside the interval $\left[U_{\min }, U_{\max }\right]$. The external knots may be set empirically to give the B-spline model a required extrapolation capability. In fact, since no data appears outside $\left[U_{\min }, U_{\max }\right]$, the exact choice of these external knots does not really matter, in terms of modeling accuracy. Also note that for QAM signals, the distribution of $x_{\mathrm{s}}$ is naturally symmetric and, therefore, the knot sequence should be chosen to be symmetric too.

Using the tensor product between the two sets of univariate B-spline basis functions [43], $B_{r}^{\left(R, P_{o}\right)}\left(x_{R}\right)$ for $1 \leq r \leq N_{R}$ and $B_{i}^{\left(I, P_{o}\right)}\left(x_{I}\right)$ for $1 \leq i \leq N_{I}$, a set of new B-spline basis functions $B_{r, i}^{\left(P_{o}\right)}(x)=\bar{B}_{r}^{\left(R, P_{o}\right)}\left(x_{R}\right) B_{i}^{\left(I, P_{o}\right)}\left(x_{I}\right)$ for $1 \leq r \leq N_{R}$ and $1 \leq i \leq N_{I}$, defined on $x=x_{R}+\mathrm{j} x_{I}$, can be formed and used to yield the complex-valued B-spline neural network

$$
\begin{aligned}
\widehat{w}=\widehat{\Psi}(x) & =\sum_{r=1}^{N_{R}} \sum_{i=1}^{N_{I}} B_{r, i}^{\left(P_{o}\right)}(x) \theta_{r, i} \\
& =\sum_{r=1}^{N_{R}} \sum_{i=1}^{N_{I}} B_{r}^{\left(R, P_{o}\right)}\left(x_{R}\right) B_{i}^{\left(I, P_{o}\right)}\left(x_{I}\right) \theta_{r, i},
\end{aligned}
$$

where $\theta_{r, i}=\theta_{r, i_{R}}+\mathrm{j} \theta_{r, i_{I}} \in \mathbb{C}, 1 \leq r \leq N_{R}$ and $1 \leq i \leq$ $N_{I}$, are the weights. Denote the weight vector of the B-spline model (15) as

$$
\boldsymbol{\theta}=\left[\begin{array}{lll}
\theta_{1,1} & \theta_{1,2} \cdots \theta_{r, i} \cdots \theta_{N_{R}, N_{I}}
\end{array}\right]^{\mathrm{T}} \in \mathbb{C}^{N_{B}},
$$

where $N_{B}=N_{R} N_{I}$. The task of identifying the nonlinearity $\Psi(\cdot)$ is turned into one of estimating the parameter vector $\boldsymbol{\theta}$.

Remark 2: A traditional way of modeling a nonlinearity in the univariate dimension of $x_{\mathrm{s}} \in \mathbb{R}$ is to use a univariate polynomial model with the polynomial degree $P_{o}$, which has $P_{o}+1$ basis functions given by

$$
1, x_{\mathrm{s}}, x_{\mathrm{s}}^{2}, \cdots, x_{\mathrm{s}}^{P_{o}} .
$$

Thus, the tensor-product polynomial model for modeling the complex-valued nonlinearity $\Psi$ has $\left(P_{o}+1\right)^{2}$ basis functions for any given input $x \in \mathbb{C}$, and the complexity of the polynomial model is on the order of $\mathrm{O}\left(\left(P_{o}+1\right)^{2}\right)$. Although the complex-valued B-spline model (15) has $N_{\mathrm{s}}^{2}$ basis functions, where $N_{\mathrm{s}}=N_{R}=N_{I}$, no more than $\left(P_{o}+1\right)^{2}$ of them are nonzero for any given $x \in \mathbb{C}$. Therefore, the complexity of the B-spline model (15) is also on the order of $\mathrm{O}\left(\left(P_{o}+1\right)^{2}\right)$. The details of this B-spline model's complexity can be found in [45]-[50].

The biggest advantage of using the B-spline model, rather than the polynomial model, is however that B-spline basis functions are optimally stable bases and they have the maximum numerical robustness [51]-[53]. This optimal robustness property of the B-spline model is due to the convexity of its model bases, i.e., they are all positive and sum to one. To illustrate this optimality of the B-spline model in comparison with the polynomial model, consider the univariate nonlinearity that can be represented by the polynomial model of degree $P_{o}$ exactly as

$$
y_{\mathrm{s}}=\sum_{i=0}^{P_{o}} a_{i} x_{\mathrm{s}}^{i}
$$

as well as by the following B-spline model exactly as

$$
y_{\mathrm{s}}=\sum_{i=1}^{N_{\mathrm{s}}} b_{i} B_{i}^{\left(\mathrm{s}, P_{o}\right)}\left(x_{\mathrm{s}}\right) \text {. }
$$

Because of the noisy training data, the estimated model coefficients are perturbed from their true values to $\widehat{a}_{i}=a_{i}+\varepsilon_{i}$ for the polynomial model, and to $\widehat{b}_{i}=b_{i}+\varepsilon_{i}$ for the B-spline model. Assume that all the estimation noises $\varepsilon_{i}$ are bounded by $\left|\varepsilon_{i}\right|<\varepsilon_{\max }$. The upper bound of $\left|y_{\mathrm{s}}-\widehat{y}_{\mathrm{s}}\right|$ for the B-spline model is given by

$$
\begin{aligned}
\left|y_{\mathrm{s}}-\widehat{y}_{\mathrm{s}}\right| & =\left|\sum_{i=1}^{N_{\mathrm{s}}} b_{i} B_{i}^{\left(\mathrm{s}, P_{o}\right)}\left(x_{\mathrm{s}}\right)-\sum_{i=1}^{N_{\mathrm{s}}} \widehat{b}_{i} B_{i}^{\left(\mathrm{s}, P_{o}\right)}\left(x_{\mathrm{s}}\right)\right| \\
& <\varepsilon_{\max }\left|\sum_{i=1}^{N_{\mathrm{s}}} B_{i}^{\left(\mathrm{s}, P_{o}\right)}\left(x_{\mathrm{s}}\right)\right|=\varepsilon_{\max } .
\end{aligned}
$$

This upper bound of the B-spline model output perturbation only depends on the upper bound of the perturbation noise, and it does not depend on the input value $x_{\mathrm{s}}$, the number of basis functions $N_{\mathrm{s}}$ or the polynomial degree $P_{o}$. Hence, the B-spline model enjoys the maximum numerical robustness. By contrast, the upper bound of $\left|y_{\mathrm{s}}-\widehat{y}_{\mathrm{s}}\right|$ for the polynomial model is given by

$$
\left|y_{\mathrm{s}}-\widehat{y}_{\mathrm{s}}\right|=\left|\sum_{i=0}^{P_{o}} a_{i} x_{\mathrm{s}}^{i}-\sum_{i=0}^{P_{o}} \widehat{a}_{i} x_{\mathrm{s}}^{i}\right|<\varepsilon_{\max }\left|\sum_{i=0}^{P_{o}} x_{\mathrm{s}}^{i}\right| .
$$

The upper bound of the polynomial model output perturbation depends not only on the upper bound of the perturbation noise but also on the input value $x_{\mathrm{s}}$ and the polynomial degree $P_{o}$. The higher the polynomial degree $P_{o}$, the more serious the polynomial model may be perturbed. A simple example can be found in [45], [48], [49] which demonstrates clearly the excellent numerical stability of the B-spline model over the polynomial model. Furthermore, in our previous works for nonlinear equalization of SISO Hammerstein channels [46][50], the B-spline parameterization approach clearly outperforms the polynomial parameterization approach. Therefore, in our application to nonlinear multiuser detection for MIMO 
Hammerstein channels, we will only consider the B-spline parameterization approach.

\section{B. Identification of nonlinear MIMO uplink model}

The identification of the nonlinear MIMO uplink involves

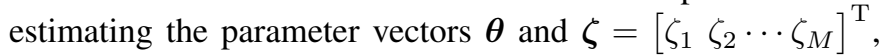
where $\zeta_{1}=1$, of the $M$ complex-valued B-spline neural networks that represent the $M$ nonlinear HPAs as well as the MIMO channel matrix $\boldsymbol{H}$, where $h_{1, m}=1$ for $1 \leq m \leq M$, based on a block of $K$ training data, $\{\boldsymbol{x}(k), \boldsymbol{y}(k)\}_{k=1}^{K}$. The outputs $\widehat{y}_{l}(k)$ of our nonlinear model for modeling the desired outputs $y_{l}(k)$ for $1 \leq l \leq L$ can be expressed by

$$
\begin{aligned}
\widehat{y}_{l}(k) & =\sum_{m=1}^{M} h_{l, m} \widehat{w}_{m}(k) \\
& =\sum_{m=1}^{M} h_{l, m} \sum_{r=1}^{N_{R}} \sum_{i=1}^{N_{I}} B_{r, i}^{\left(P_{o}\right)}\left(x_{m}(k)\right) \zeta_{m} \theta_{r, i} .
\end{aligned}
$$

Observe that the parameters to be estimated enter the model in the nonlinear triple product form of $h_{l, m} \zeta_{m} \theta_{r, i}$. Fixing one set of parameters, e.g., $h_{l, m}$, the model is still nonlinear in the other two sets of parameters. Therefore, the single-loop two-stage ALS estimation procedure of [45]-[50] cannot be extended to this case, because this single-loop two-stage ALS estimator only works for the model with bilinear parameters. We propose a new TL-3S-ALS procedure to estimate $\boldsymbol{\theta}$ and $\boldsymbol{\zeta}$ as well as $\boldsymbol{H}$. Benefiting from our unique parameterization of the nonlinear MIMO uplink, this TL-3S-ALS algorithm guarantees to obtain a unique estimate of the nonlinear MIMO uplink model.

Specifically, denote the desired output matrix $\boldsymbol{Y} \in \mathbb{C}^{L \times K}$

$$
\boldsymbol{Y}=\left[\begin{array}{cccc}
y_{1}(1) & y_{1}(2) & \cdots & y_{1}(K) \\
y_{2}(1) & y_{2}(2) & \cdots & y_{2}(K) \\
\vdots & \vdots & \cdots & \vdots \\
y_{L}(1) & y_{L}(2) & \cdots & y_{L}(K)
\end{array}\right]=\left[\begin{array}{c}
\boldsymbol{y}_{1}^{\mathrm{T}} \\
\boldsymbol{y}_{2}^{\mathrm{T}} \\
\vdots \\
\boldsymbol{y}_{L}^{\mathrm{T}}
\end{array}\right]
$$

which can be expressed as

$$
\boldsymbol{Y}=\boldsymbol{H} \boldsymbol{Q}+\boldsymbol{N},
$$

where $N \in \mathbb{C}^{L \times K}$ is the corresponding channel AWGN matrix and the regression matrix $\boldsymbol{Q} \in \mathbb{C}^{M \times K}$ is given by

$$
\boldsymbol{Q}=\left[\begin{array}{cccc}
\widehat{w}_{1}(1) & \widehat{w}_{1}(2) & \cdots & \widehat{w}_{1}(K) \\
\widehat{w}_{2}(1) & \widehat{w}_{2}(2) & \cdots & \widehat{w}_{2}(K) \\
\vdots & \vdots & \cdots & \vdots \\
\widehat{w}_{M}(1) & \widehat{w}_{M}(2) & \cdots & \widehat{w}_{M}(K)
\end{array}\right],
$$

in which

$$
\widehat{w}_{m}(k)=\sum_{r=1}^{N_{R}} \sum_{i=1}^{N_{I}} B_{r, i}^{\left(P_{o}\right)}\left(x_{m}(k)\right) \zeta_{m} \theta_{r, i} .
$$

Observe that $\boldsymbol{Q}$ is nonlinear in $\zeta_{m} \theta_{r, i}$, which is the reason why the algorithm of [45]-[50] cannot be applied.

On the other hand, the desired output vectors $\boldsymbol{y}_{l} \in \mathbb{C}^{K}$ for $1 \leq l \leq L$ can be expressed as

$$
\boldsymbol{y}_{l}=\boldsymbol{P}_{l} \boldsymbol{\theta}+\boldsymbol{n}_{l}=\boldsymbol{S}_{l} \boldsymbol{\zeta}+\boldsymbol{n}_{l},
$$

where $\boldsymbol{n}_{l} \in \mathbb{C}^{K}$ is the corresponding channel AWGN vector, and the regression matrix $\boldsymbol{P}_{l} \in \mathbb{C}^{K \times N_{B}}$ is given by

$$
\boldsymbol{P}_{l}=\left[\begin{array}{cccc}
\phi_{1,1}^{(l)}(1) & \phi_{1,2}^{(l)}(1) & \cdots & \phi_{N_{R}, N_{I}}^{(l)}(1) \\
\phi_{1,1}^{(l)}(2) & \phi_{1,2}^{(l)}(2) & \cdots & \phi_{N_{R}, N_{I}}^{(l)}(2) \\
\vdots & \vdots & \cdots & \vdots \\
\phi_{1,1}^{(l)}(K) & \phi_{1,2}^{(l)}(K) & \cdots & \phi_{N_{R}, N_{I}}^{(l)}(K)
\end{array}\right],
$$

with

$$
\begin{array}{r}
\phi_{r, i}^{(l)}(k)=\sum_{m=1}^{M} h_{l, m} \zeta_{m} B_{r, i}^{\left(P_{o}\right)}\left(x_{m}(k)\right), \\
1 \leq r \leq N_{R}, 1 \leq i \leq N_{I},
\end{array}
$$

while the regression matrix $\boldsymbol{S}_{l} \in \mathbb{C}^{K \times M}$ is given by

$$
\boldsymbol{S}_{l}=\left[\begin{array}{cccc}
h_{l, 1} \psi_{1}(1) & h_{l, 2} \psi_{2}(1) & \cdots & h_{l, M} \psi_{M}(1) \\
h_{l, 1} \psi_{1}(2) & h_{l, 2} \psi_{2}(2) & \cdots & h_{l, M} \psi_{M}(2) \\
\vdots & \vdots & \cdots & \vdots \\
h_{l, 1} \psi_{1}(k) & h_{l, 2} \psi_{2}(k) & \cdots & h_{l, M} \psi_{M}(k)
\end{array}\right],
$$

with

$$
\psi_{m}(k)=\sum_{r=1}^{N_{R}} \sum_{i=1}^{N_{I}} B_{r, i}^{\left(P_{o}\right)}\left(x_{m}(k)\right) \theta_{r, i}, 1 \leq m \leq M .
$$

Hence we have

$$
\sum_{l=1}^{L} \boldsymbol{y}_{l}=\sum_{l=1}^{L} \boldsymbol{P}_{l} \boldsymbol{\theta}+\sum_{l=1}^{L} \boldsymbol{n}_{l}=\sum_{l=1}^{L} \boldsymbol{S}_{l} \boldsymbol{\zeta}+\sum_{l=1}^{L} \boldsymbol{n}_{l},
$$

or

$$
\overline{\boldsymbol{y}}=\overline{\boldsymbol{P}} \boldsymbol{\theta}+\overline{\boldsymbol{n}}=\overline{\boldsymbol{S}} \boldsymbol{\zeta}+\overline{\boldsymbol{n}} .
$$

Observe that we can express the model as linear in $\boldsymbol{H}$ but the corresponding regression matrix is bilinear in $\theta$ and $\zeta$. On the other hand, if we express the model as linear in $\boldsymbol{\theta}$, the resulting regression matrix is bilinear in $\boldsymbol{H}$ and $\zeta$. Similarly, when we express the model as linear in $\zeta$, the regression matrix is bilinear in $\boldsymbol{H}$ and $\boldsymbol{\theta}$. We propose the following TL3S-ALS procedure to jointly estimate $\boldsymbol{H}, \boldsymbol{\theta}$ and $\boldsymbol{\zeta}$.

Initialization. Replacing $\widehat{w}_{m}(k)$ with $x_{m}(k)$ in (25), we have the 'LS' estimate of $\boldsymbol{H}$, denoted as $\widehat{\boldsymbol{H}}^{[0]}$, given by

$$
\widehat{\boldsymbol{H}}^{[0]}=\boldsymbol{Y} \boldsymbol{X}^{\mathrm{H}}\left(\boldsymbol{X} \boldsymbol{X}^{\mathrm{H}}\right)^{-1},
$$

where $\boldsymbol{X} \in \mathbb{C}^{M \times K}$ is the 'regression matrix' given by

$$
\boldsymbol{X}=\left[\begin{array}{cccc}
x_{1}(1) & x_{1}(2) & \cdots & x_{1}(K) \\
x_{2}(1) & x_{2}(2) & \cdots & x_{2}(K) \\
\vdots & \vdots & \cdots & \vdots \\
x_{M}(1) & x_{M}(2) & \cdots & x_{M}(K)
\end{array}\right]
$$

Each column of $\widehat{\boldsymbol{H}}^{[0]}$ is then divided by its first-row element, namely,

$$
\widehat{h}_{l, m}^{[0]}=\widehat{h}_{l, m}^{[0]} / \widehat{h}_{1, m}^{[0]}, 1 \leq l \leq L, 1 \leq m \leq M .
$$

Also initialize $\zeta^{[0]}$ with $\zeta_{m}^{[0]}=1$ for $1 \leq m \leq M$.

TL-3S-ALS estimator. For $1 \leq \tau \leq \tau_{\max }$, where $\tau_{\max }$ is the maximum number of iterations in the outer loop, perform:

a) Estimating the parameter vectors of the MHPAs. Set $\boldsymbol{H}$ to $\widehat{\boldsymbol{H}}^{[\tau-1]}$ in $\overline{\boldsymbol{P}}$ and $\overline{\boldsymbol{S}}$. For $1 \leq \iota \leq \iota_{\max }$, where $\iota_{\max }$ is the 
maximum number of iterations for stage a) or the inner loop, and with $\zeta^{[0}=\boldsymbol{\zeta}^{[\tau-1]}$, perform:

a.1) Set $\boldsymbol{\zeta}$ to $\boldsymbol{\zeta}^{[\iota-1}$ in $\overline{\boldsymbol{P}}$ and denote the resultant regression matrix as $\overline{\boldsymbol{P}}^{[\iota}$. Then the LS estimate of $\boldsymbol{\theta}$ is readily be given by

$$
\boldsymbol{\theta}^{[\iota}=\left(\left(\overline{\boldsymbol{P}}^{[\iota}\right)^{\mathrm{H}} \overline{\boldsymbol{P}}^{[\iota}+\lambda \boldsymbol{I}_{N_{B}}\right)^{-1}\left(\overline{\boldsymbol{P}}^{[\iota}\right)^{\mathrm{H}} \overline{\boldsymbol{y}},
$$

where $\lambda$ is a very small regularization parameter, e.g., $\lambda=$ $10^{-6}$.

a.2) Set $\boldsymbol{\theta}$ to $\boldsymbol{\theta}^{[\iota}$ in $\overline{\boldsymbol{S}}$ and denote the resultant regression matrix as $\bar{S}^{[\iota}$. Then the LS estimate of $\zeta$ is readily be given by

$$
\boldsymbol{\zeta}^{[\iota}=\left(\left(\overline{\boldsymbol{S}}^{[\iota}\right)^{\mathrm{H}} \overline{\boldsymbol{S}}^{[\iota}\right)^{-1}\left(\overline{\boldsymbol{S}}^{[\iota}\right)^{\mathrm{H}} \overline{\boldsymbol{y}} .
$$

Then normalize $\boldsymbol{\zeta}^{[\iota}$ with

$$
\zeta_{m}^{[\iota}=\zeta_{m}^{[\iota} / \zeta_{1}^{[\iota}, 1 \leq m \leq M .
$$

At the end of stage a), we have $\boldsymbol{\theta}^{[\tau]}=\boldsymbol{\theta}^{\left[\iota_{\max }\right.}$ and $\boldsymbol{\zeta}^{[\tau]}=$ $\zeta^{\left[\iota_{\max }\right.}$.

b) Estimating the MIMO channel matrix. Set $\boldsymbol{\theta}=\boldsymbol{\theta}^{[\tau]}$ and $\zeta=\zeta^{[\tau]}$ in $\boldsymbol{Q}$ and denote the resultant regression matrix as $\boldsymbol{Q}^{[\tau]}$. The LS estimate of $\boldsymbol{H}$ is given by

$$
\widehat{\boldsymbol{H}}^{[\tau]}=\boldsymbol{Y}\left(\boldsymbol{Q}^{[\tau]}\right)^{\mathrm{H}}\left(\boldsymbol{Q}^{[\tau]}\left(\boldsymbol{Q}^{[\tau]}\right)^{\mathrm{H}}\right)^{-1},
$$

which is followed by the following operation

$$
\widehat{h}_{l, m}^{[\tau]}=\widehat{h}_{l, m}^{[\tau]} / \widehat{h}_{1, m}^{[\tau]}, 1 \leq l \leq L, 1 \leq m \leq M .
$$

Remark 3: Observe that this identification procedure involves the two loops: the outer loop of a) and b) as well as the inner loop of a.1) and a.2), with the three stages of alternating 'LS' estimation: (37), (38) and (40). The maximum number of the outer-loop iterations $\tau_{\max }=1$ or 2 is sufficient. This is because the initial LS estimate $\widehat{\boldsymbol{H}}^{[0]}$ of (34) is an 'unbiased' estimate of $\boldsymbol{H}$ scaled by the HPAs' complex-valued gain. Therefore, the 'normalized' $\widehat{\boldsymbol{H}}^{[0]}$ of (36) is an accurate estimate of $\boldsymbol{H}$ in (8). Thus, $\tau_{\max }=1$ is in fact sufficient. Given an accurate estimate of $\boldsymbol{H}$, the inner loop a) of the two-stage ALS estimator converges to the unique estimates of $\boldsymbol{\theta}$ and $\boldsymbol{\zeta}$ very fast, owing to the unique parameterization of the HPAs and the closed-form LS estimates of (37) and (38). In fact, the maximum number of the inner iterations $\iota_{\max }=2$ to 4 is sufficient.

\section{Identification of inverse nonlinear mappings of HPAs}

As shown in Section II, to implementing the multiuser detection for the nonlinear MIMO uplink, we also requires the inverse mappings of the $M$ HPAs defined by

$$
x_{m}(k)=\left(\zeta_{m} \Psi\right)^{-1}\left(w_{m}(k)\right)=\Phi_{m}\left(w_{m}(k)\right), 1 \leq m \leq M .
$$

We utilise another complex-valued B-spline neural network to model $\Phi_{m}(\cdot)$. Define the two knots sequences similar to (11) for the real and imaginary parts of $w_{m}$, respectively. Similar to (14), we construct the inverting B-spline neural network ${ }^{1}$

$$
\begin{aligned}
\widehat{x}_{m} & =\widehat{\Phi}_{m}\left(w_{m}\right)=\sum_{r=1}^{N_{R}} \sum_{i=1}^{N_{I}} B_{r, i}^{\left(P_{o}\right)}\left(w_{m}\right) \alpha_{r, i}^{(m)} \\
& =\sum_{r=1}^{N_{R}} \sum_{i=1}^{N_{I}} B_{r}^{\left(R, P_{o}\right)}\left(w_{m_{R}}\right) B_{i}^{\left(I, P_{o}\right)}\left(w_{m_{I}}\right) \alpha_{r, i}^{(m)},
\end{aligned}
$$

where $B_{r}^{\left(R, P_{o}\right)}\left(w_{m_{R}}\right)$ and $B_{i}^{\left(I, P_{o}\right)}\left(w_{m_{I}}\right)$ are respectively calculated according to the De Boor recursion (13) and (14), while

$$
\boldsymbol{\alpha}^{(m)}=\left[\begin{array}{lll}
\alpha_{1,1}^{(m)} & \alpha_{1,2}^{(m)} \cdots \alpha_{r, i}^{(m)} \cdots \alpha_{N_{R}, N_{I}}^{(m)}
\end{array}\right]^{\mathrm{T}} \in \mathbb{C}^{N_{B}}
$$

is the parameter vector of this inverting B-spline neural network. Thus, the task of inverting the nonlinear HPA $\zeta_{m} \Psi($ ) becomes one of estimating $\boldsymbol{\alpha}^{(m)}$.

To estimate $\boldsymbol{\alpha}^{(m)}$ requires the input-output training data $\left\{w_{m}(k), x_{m}(k)\right\}_{k=1}^{K}$ but $w_{m}(k)$ is unobserved and therefore unavailable for this modeling. Following the same approach of [45]-[50], the pseudo training data $\left\{\widehat{\bar{w}}_{m}(k), x_{m}(k)\right\}_{k=1}^{K}$ are constructed as a byproduct of the nonlinear MIMO channel identification. Specifically, given the estimated HPA's nonlinearity $\widehat{\zeta}_{m} \widehat{\Psi}(\cdot)$, we calculate $\widehat{\bar{w}}_{m}(k)=\widehat{\zeta}_{m} \widehat{\Psi}\left(x_{m}(k)\right)$ to substitute for $w_{m}(k)$ as the training input. This allows us to estimate the inverting model $\Phi_{m}(\cdot)$ based on the LS method. However, the training input $\widehat{\bar{w}}_{m}(k)$ is highly noisy and this may introduce potentially serious bias in the estimate.

Over the pseudo training data set $\left\{\widehat{\bar{w}}_{m}(k), x_{m}(k)\right\}_{k=1}^{K}$, we form the regression matrix $\widetilde{\mathbf{B}}_{m} \in \mathbb{R}^{K \times N_{B}}$

$$
\widetilde{\boldsymbol{B}}_{m}=\left[\begin{array}{ccc}
B_{1,1}^{\left(P_{o}\right)}\left(\widehat{\bar{w}}_{m}(1)\right) & \cdots & B_{N_{R}, N_{I}}^{\left(P_{o}\right)}\left(\widehat{\bar{w}}_{m}(1)\right) \\
B_{1,1}^{\left(P_{o}\right)}\left(\widehat{\bar{w}}_{m}(2)\right) & \cdots & B_{N_{R}, N_{I}}^{\left(P_{o}\right.}\left(\widehat{\bar{w}}_{m}(2)\right) \\
\vdots & \cdots & \vdots \\
B_{1,1}^{\left(P_{o}\right)}\left(\widehat{\bar{w}}_{m}(K)\right) & \cdots & B_{N_{R}, N_{I}}^{\left(P_{o}\right)}\left(\widehat{\bar{w}}_{m}(K)\right)
\end{array}\right]
$$

and the desired output vector

$$
\boldsymbol{x}_{m}=\left[x_{m}(1) x_{m}(2) \cdots x_{m}(K)\right]^{\mathrm{T}} .
$$

Then the closed-form LS estimate $\boldsymbol{\alpha}^{(m)}$ is readily given by

$$
\widehat{\boldsymbol{\alpha}}^{(m)}=\left(\widetilde{\boldsymbol{B}}_{m}^{\mathrm{T}} \widetilde{\boldsymbol{B}}_{m}\right)^{-1} \widetilde{\boldsymbol{B}}_{m}^{\mathrm{T}} \boldsymbol{x}_{m} .
$$

Remark 4: A tensor-product polynomial model, having a polynomial degree of $P_{o}$ in each dimension, can also be utilized to estimate $\left(\zeta_{m} \Psi\right)^{-1}(\cdot)$, based on the same LS identification procedure developed here. It is well-known that since the input $\widehat{\bar{w}}_{m}(k)$ is noisy, the LS estimate (47) is generally biased. Thus, the maximum robustness property of the B-spline model as discussed in Remark 2 is particularly helpful in minimizing this bias. Consequently, the B-spline based approach yields significantly better performance than the polynomial based approach, and this has been confirmed in our previous studies for nonlinear equalization of SISO Hammerstein channels [46]-[50]. For this reason, we will only consider the B-spline inversion in our application to nonlinear multiuser detection for nonlinear MIMO uplink.

\footnotetext{
${ }^{1}$ We assume that the same number of basis functions and polynomial degree are used for the two B-spline neural networks $\Psi(x)$ and $\Phi_{m}\left(w_{m}\right)$.
} 
TABLE I

EMPIRICALLY DETERMINED KNOT SEQUENCES.

\begin{tabular}{l}
\hline Knot sequence for $x_{R}$ and $x_{I}$ \\
$-10.0,-9.0,-1.0,-\mathbf{0 . 9},-0.05,-0.02,0.0,0.02,0.05, \mathbf{0 . 9}, 1.0,9.0,10.0$ \\
\hline Knot sequence for $w_{R}$ and $w_{I}$ \\
$-20.0,-18.0,-3.0,-\mathbf{1 . 4},-0.8,-0.4,0.0,0.4,0.8, \mathbf{1 . 4}, 3.0,18.0,20.0$ \\
\hline
\end{tabular}

\section{Simulation Study}

\section{A. Simulation system set up}

The BS is equipped with $L=4$ antennas to support $M=$ 3 single-antenna MUs. The 64-QAM signaling is employed, and the HPA deployed in an MU's transmitter is described by (3) and (4). The parameters of this nonlinear HPA are set to those of the NEC GaAs power amplifier adopted in the recent wireless standards [30], [31], which are

$$
\begin{aligned}
& g_{a}=19, \beta_{a}=0.81, A_{\mathrm{sat}}=1.4 ; \alpha_{\phi}=-48000, \\
& \beta_{\phi}=0.123, q_{1}=3.8, q_{2}=3.7 .
\end{aligned}
$$

Since we have $L$ receive antennas and $M$ users, the MIMO system's average signal-to-noise ratio (SNR) is defined as

$$
\text { Average SNR }=\frac{\sum_{m=1}^{M} \sigma_{w_{m}}^{2}}{L \cdot 2 \sigma_{n}^{2}}
$$

where $\sigma_{w_{m}}^{2}=E\left\{\left|w_{m}(k)\right|^{2}\right\}$ is the average power of the $m$ th MU's transmit signal. The number of training samples is set to $K=1000$. We also repeat the identification with $K=500$, and the results obtained are practically identical to those obtained with $K=1000$. The number of B-spline basis functions is chosen to be $N_{R}=N_{I}=8$ and the polynomial degree is set to $P_{o}=4$. The empirically determined knot

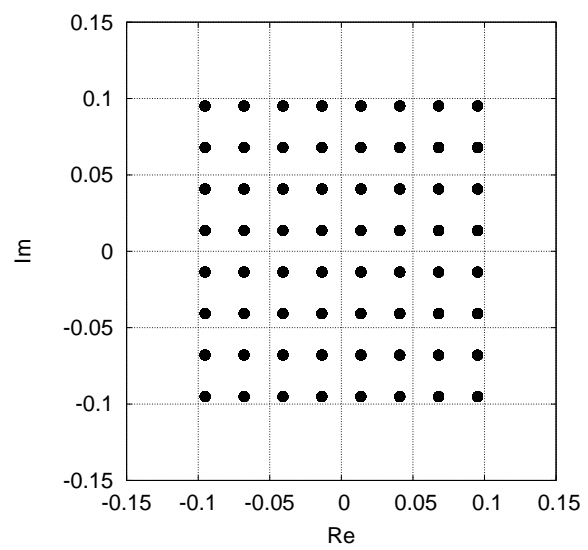

Fig. 2. 64-QAM constellation employed by MUs.

sequences covering the HPA's operating range are listed in Table I. Referring to Fig. 2, owing to the symmetric distribution of $x_{R}$ and $x_{I}$, the knot sequences for $x_{R}$ and $x_{I}$ are identical. Also observe that how the internal knots are placed within the input signal range. Similarly, the knot sequences for $w_{R}$ and $w_{I}$ are identical, and they are chosen according to the range of the HPA output. The number of outer iterations for the TL-3S-ALS estimator is set to $\tau_{\max }=2$, and its inner iteration number is set to $\iota_{\max }=2$.

\section{B. Estimation results}

First, we consider the true MIMO channel matrix $\boldsymbol{H}$ as given in Table II. The HPAs' OBO is $3 \mathrm{~dB}$ and the system's average SNR is $25 \mathrm{~dB}$. The B-spline based identification and detection scheme presented in Section III is applied to this nonlinear MIMO uplink. The results are obtained over

TABLE II

\begin{tabular}{|c|c|c|}
\hline \multicolumn{3}{|c|}{ True MIMO channel matrix $\boldsymbol{H}=\left[h_{l, m}\right]$} \\
\hline 1 & 1 & 1 \\
\hline$-0.5690-\mathrm{j} 0.2982$ & $0.3755+\mathrm{j} 0.4018$ & $1.6995-\mathrm{j} 0.2905$ \\
\hline $1.3457-\mathrm{j} 0.3477$ & $-0.1295-\mathrm{j} 1.4125$ & $-0.5323-\mathrm{j} 0.4941$ \\
\hline $0.3291-\mathrm{j} 0.1268$ & $1.0269+\mathrm{j} 0.4665$ & $-0.5798+\mathrm{j} 0.8334$ \\
\hline \multicolumn{3}{|c|}{ True weightings of MUs' HPAs $\zeta$} \\
\hline 1 & 1 & 1 \\
\hline \multicolumn{3}{|c|}{ Estimated MIMO channel matrix $\widehat{\boldsymbol{H}}=\left|\widehat{h}_{l, m}\right|$ by B-spline approach } \\
\hline 1 & 1 & 1 \\
\hline$-0.5687-\mathrm{j} 0.2982( \pm 0.0013 \pm \mathrm{j} 0.0014)$ & $0.3756+\mathrm{j} 0.4018( \pm 0.0013 \pm \mathrm{j} 0.0014)$ & $1.6998-\mathrm{j} 0.2904( \pm 0.0022 \pm \mathrm{j} 0.0024)$ \\
\hline $1.3456-\mathrm{j} 0.3477( \pm 0.0016 \pm \mathrm{j} 0.0019)$ & $-0.1298-\mathrm{j} 1.4129( \pm 0.0021 \pm \mathrm{j} 0.0022)$ & $-0.5322-\mathrm{j} 0.4943( \pm 0.0014 \pm \mathrm{j} 0.0016)$ \\
\hline $0.3290-\mathrm{j} 0.1268( \pm 0.0013 \pm \mathrm{j} 0.0012)$ & $1.0274+\mathrm{j} 0.4663( \pm 0.0019 \pm \mathrm{j} 0.0017)$ & $-0.5797+\mathrm{j} 0.8333( \pm 0.0017 \pm \mathrm{j} 0.0018)$ \\
\hline \multicolumn{3}{|c|}{ Estimated weightings of MUs' HPAs $\widehat{\zeta}$ by B-spline approach } \\
\hline 1 & $0.9996+\mathrm{j} 0.0002( \pm 0.0015 \pm \mathrm{j} 0.0016)$ & $0.9998-\mathrm{j} 0.0000( \pm 0.0016 \pm \mathrm{j} 0.0018)$ \\
\hline \multicolumn{3}{|c|}{ Estimated MIMO channel matrix $\widehat{\boldsymbol{H}}^{[0]}=\left[\widehat{h}_{l, m}^{[0]}\right]$ by linear estimator } \\
\hline $10.4946-\mathrm{j} 0.9324( \pm 0.0736 \pm \mathrm{j} 0.0582)$ & $10.4888-\mathrm{j} 0.9450( \pm 0.0867 \pm \mathrm{j} 0.0580)$ & $10.4900-\mathrm{j} 0.9393( \pm 0.0794 \pm \mathrm{j} 0.0516)$ \\
\hline$-6.2475-\mathrm{j} 2.5743( \pm 0.0703 \pm \mathrm{j} 0.0693)$ & $4.3207+\mathrm{j} 3.8563( \pm 0.0691 \pm \mathrm{j} 0.0745)$ & $17.5497-\mathrm{j} 4.6481( \pm 0.1046 \pm \mathrm{j} 0.0342)$ \\
\hline $13.8010-\mathrm{j} 4.9279( \pm 0.0963 \pm \mathrm{j} 0.0554)$ & $-2.6945-\mathrm{j} 14.6925( \pm 0.0645 \pm \mathrm{j} 0.1032)$ & $-6.0389-\mathrm{j} 4.6895( \pm 0.0813 \pm \mathrm{j} 0.0809)$ \\
\hline $3.3240-\mathrm{j} 1.6411( \pm 0.0578 \pm \mathrm{j} 0.0510)$ & $11.2102+\mathrm{j} 3.9206( \pm 0.0683 \pm \mathrm{j} 0.0580)$ & $-5.2930+\mathrm{j} 9.2999( \pm 0.0554 \pm \mathrm{j} 0.0608)$ \\
\hline \multicolumn{3}{|c|}{ 'Normalized' linear estimate $\widehat{\boldsymbol{H}}^{[0]}:\left[\widehat{h}_{l, m}^{[0]} / \widehat{h}_{1, m}^{[0]}\right]$} \\
\hline 1 & 1 & 1 \\
\hline$-0.5690-\mathrm{j} 0.2959( \pm 0.0087 \pm \mathrm{j} 0.0094)$ & $0.3758+\mathrm{j} 0.4015( \pm 0.0068 \pm \mathrm{j} 0.0069)$ & $1.6990-\mathrm{j} 0.2910( \pm 0.0099 \pm \mathrm{j} 0.0087)$ \\
\hline $1.3461-\mathrm{j} 0.3500( \pm 0.0090 \pm \mathrm{j} 0.0095)$ & $-0.1297-\mathrm{j} 1.4125( \pm 0.0086 \pm \mathrm{j} 0.0071)$ & $-0.5314-\mathrm{j} 0.4946( \pm 0.0078 \pm \mathrm{j} 0.0067)$ \\
\hline $0.3280-\mathrm{j} 0.1272( \pm 0.0055 \pm \mathrm{j} 0.0053)$ & $1.0268+\mathrm{j} 0.4663( \pm 0.0074 \pm \mathrm{j} 0.0075)$ & $-0.5793+\mathrm{j} 0.8347( \pm 0.0076 \pm \mathrm{j} 0.0079)$ \\
\hline
\end{tabular}

IDENTIFICATION RESULTS FOR THE MIMO CHANNEL MATRIX $\boldsymbol{H}$ OF THE MIMO HAMMERSTEIN CHANNEL. THE OBO IS 3 DB AND THE AVERAGE SNR IS 25 DB. THE RESULTS ARE OBTAINED OVER 100 IDENTIFICATION RUNS, AND ARE PRESENTED AS: AVERAGE ESTIMATE (STANDARD DEVIATION). 

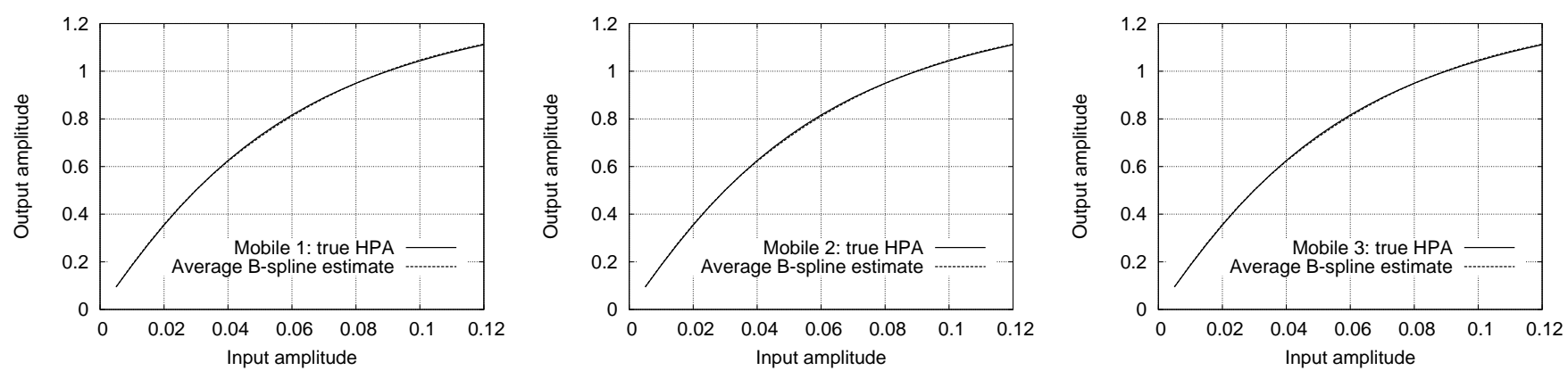

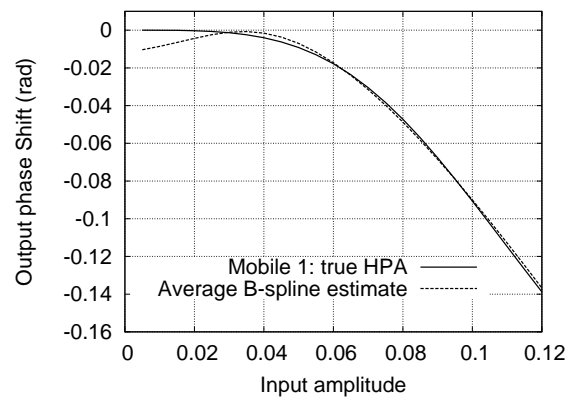

(a)

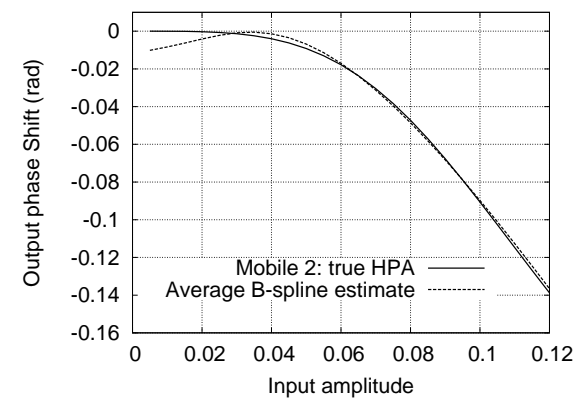

(b)

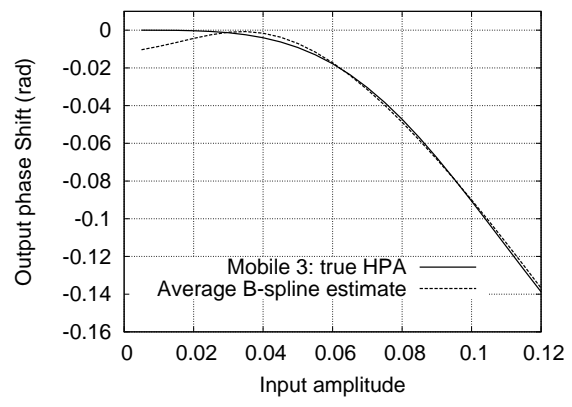

(c)

Fig. 3. Comparison of the true HPA's nonlinearity $\zeta_{m} \Psi(\cdot)$ and the B-spline estimated HPA nonlinearity $\widehat{\zeta}_{m} \widehat{\Psi}_{(\cdot)}$ averaged over 100 identification runs under $\mathrm{OBO}$ of $3 \mathrm{~dB}$ and average SNR of $25 \mathrm{~dB}$ : (a) $\mathrm{MU} m=1$, (b) $\mathrm{MU} m=2$, and (c) MU $m=3$.
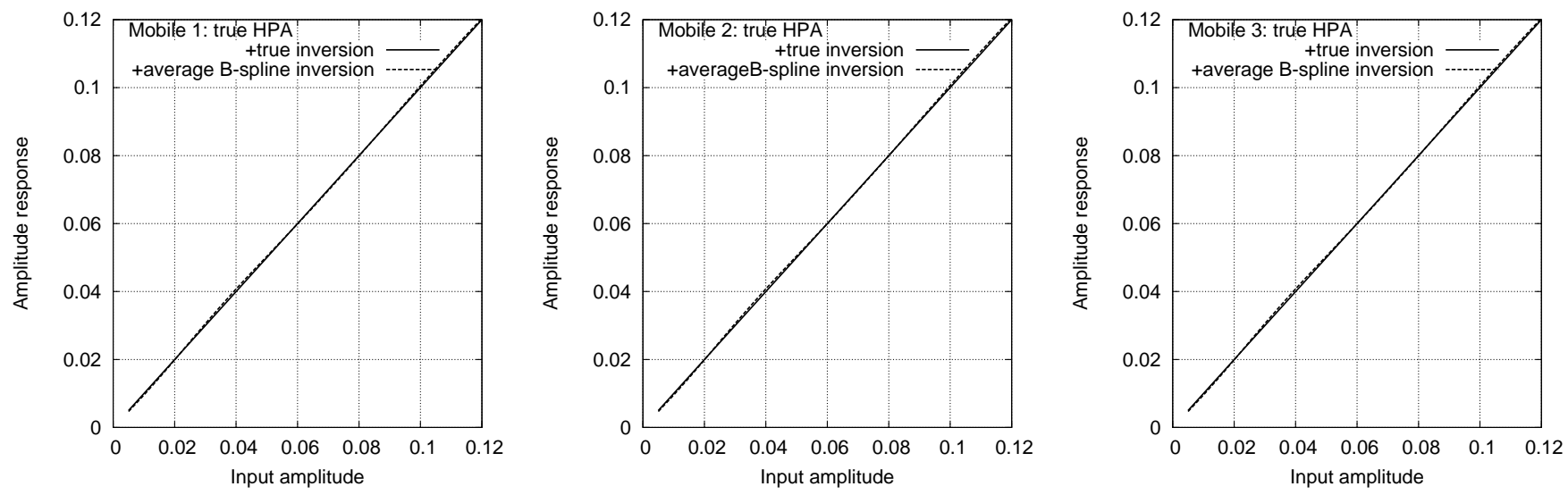

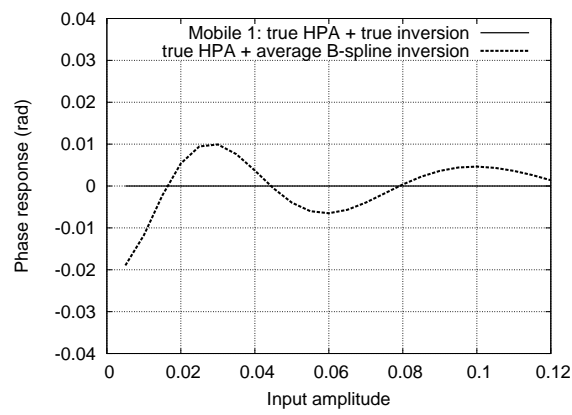

(a)

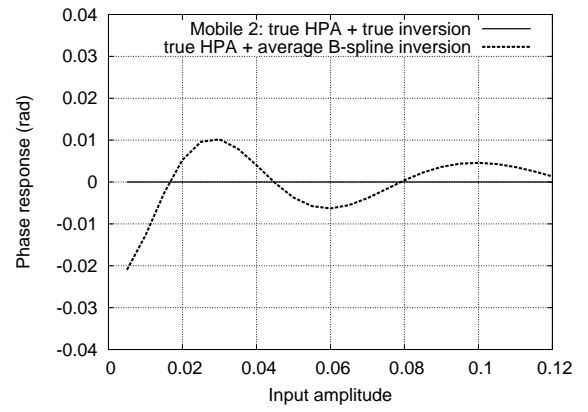

(b)

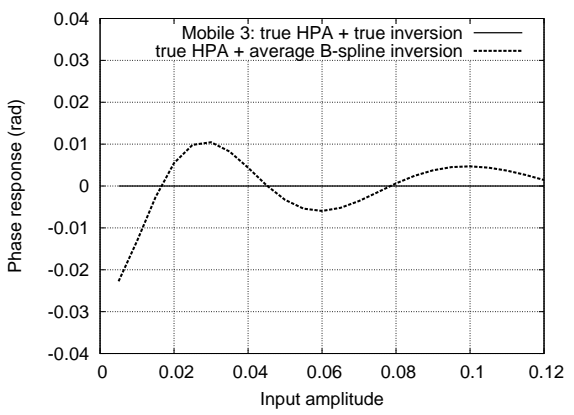

(c)

Fig. 4. Combined response of the true HPA $\zeta_{m} \Psi(\cdot)$ and the estimated B-spline inversion $\left(\widehat{\zeta}_{m} \widehat{\Psi}\right)^{-1}(\cdot)$ averaged over 100 identification runs under the $\mathrm{OBO}$ of $3 \mathrm{~dB}$ and the average SNR of $25 \mathrm{~dB}$ : (a) MU $m=1$, (b) $\mathrm{MU} m=2$, and (c) $\mathrm{MU} m=3$.

100 identification experiments and the estimation results are presented as average estimate with standard deviation.

The estimated MIMO channel matrix $\widehat{\boldsymbol{H}}$ by the B-spline based estimator is also listed in Table II, where it can be seen that $\widehat{\boldsymbol{H}}$ is a very accurate unbiased estimate of the true MIMO channel matrix $\boldsymbol{H}$ with very small estimation error standard deviations. Fig. 3 compares the B-spline estimated
HPA nonlinearity $\widehat{\zeta}_{m} \widehat{\Psi}(\cdot)$ averaged over 100 identification runs with the true HPA's nonlinearity $\zeta_{m} \Psi(\cdot)$, where $\zeta_{m}=1$ for $1 \leq m \leq 3$. Observe that the amplitude response of $\widehat{\zeta}_{m} \widehat{\Psi}(\cdot)$ closely matches the true HPA's amplitude response, and the estimation error of the phase response of $\widehat{\zeta}_{m} \widehat{\Psi}(\cdot)$ is no more than 0.01 radian. The combined responses of the HPA's true nonlinearity and its estimated inversions obtained 


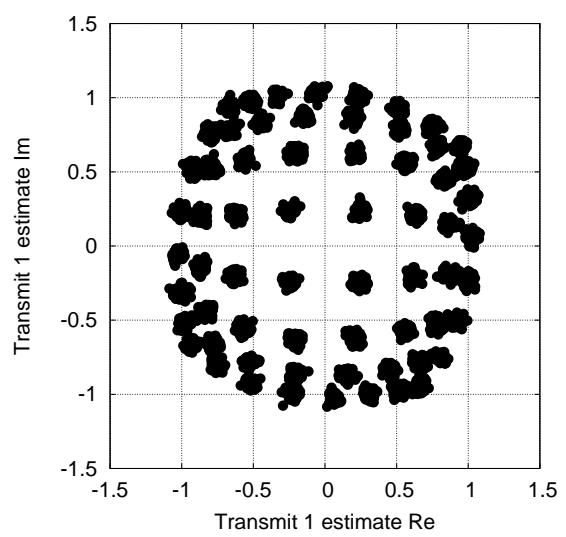

(a)

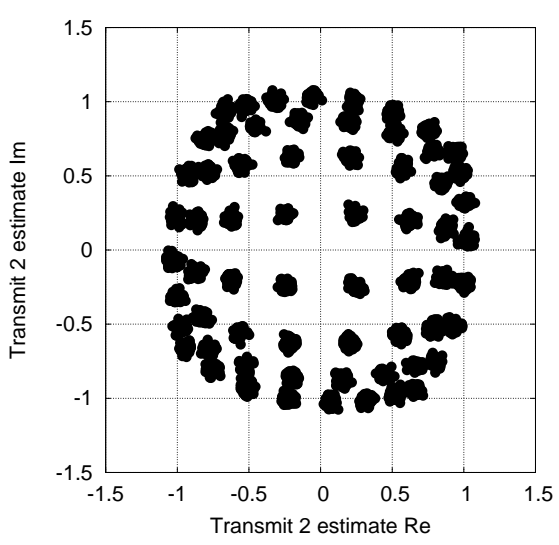

(b)

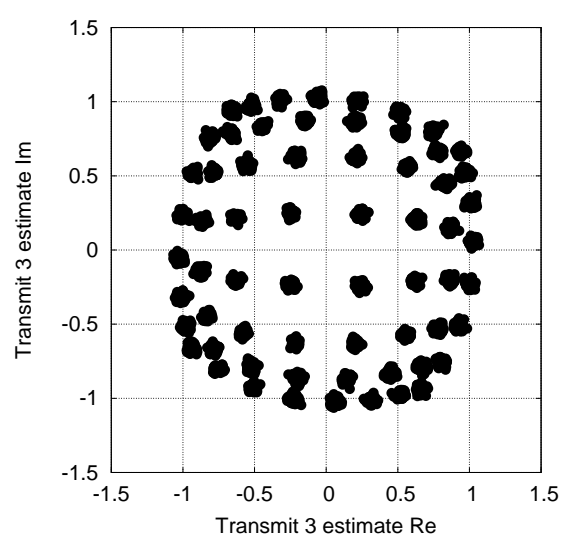

(c)

Fig. 5. ZF detection of $\boldsymbol{w}(k)$ using the estimated $\widehat{\boldsymbol{H}}$ identified by the B-spline based estimation scheme at a typical identification run under OBO of $3 \mathrm{~dB}$ and average SNR of $25 \mathrm{~dB}$ : (a) $\widehat{w}_{1}(k)$, (b) $\widehat{w}_{2}(k)$, and (c) $\widehat{w}_{3}(k)$.

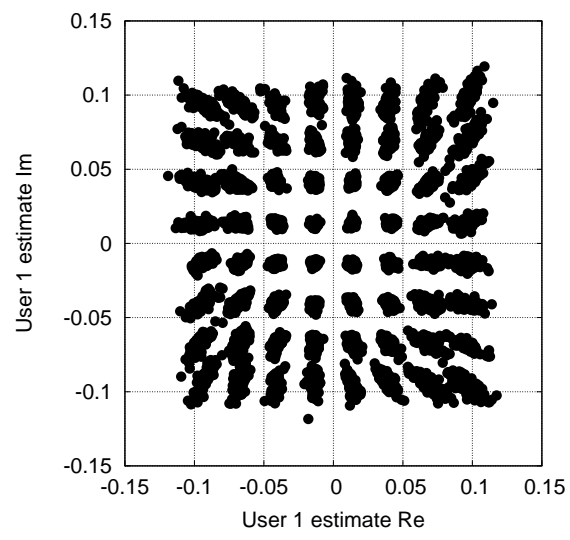

(a)

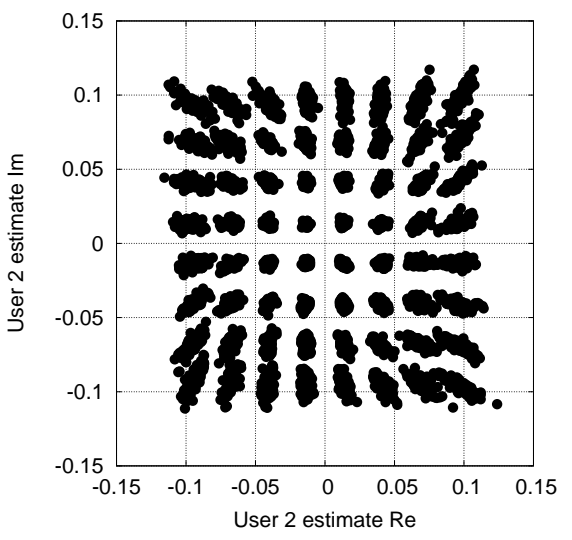

(b)

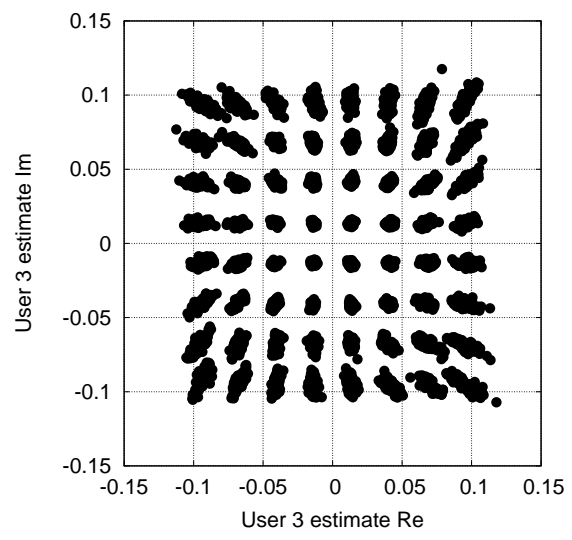

(c)

Fig. 6. Detection of $\boldsymbol{x}(k)$ using the estimated $\widehat{\boldsymbol{H}}$ and the B-spline inversions $\left(\widehat{\zeta}_{m} \widehat{\Psi}\right)^{-1}(\cdot)$ obtained by the B-spline based estimation scheme at a typical identification run under OBO of $3 \mathrm{~dB}$ and average SNR of $25 \mathrm{~dB}$ : (a) $\widehat{x}_{1}(k)$, (b) $\widehat{x}_{2}(k)$, and (c) $\widehat{x}_{3}(k)$.

by the proposed B-spline inverting scheme are illustrated in Fig. 4, where it can be seen that the combined response of the true HPA's nonlinearity $\zeta_{m} \Psi(\cdot)$ and its BS inversion estimate $\widehat{\Phi}_{m}(\cdot)=\left(\widehat{\zeta}_{m} \widehat{\Psi}\right)^{-1}(\cdot)$ satisfies

$$
\widehat{\Phi}_{m}\left(\zeta_{m} \Psi\left(x_{m}\right)\right) \approx x_{m}, 1 \leq m \leq 3 .
$$

That is, the magnitude of the combined response is $\left|\widehat{\Phi}_{m}\left(\zeta_{m} \Psi\left(x_{m}\right)\right) \approx\right| x_{m} \mid$, and the phase shift of the combined response is approximately zero. This clearly shows the accuracy of our proposed B-spline inversion scheme based on the pseudo training data only. Observe from Fig. 4 that owing to the noise in the pseudo training input, the maximum estimation error of the combined phase response increases to 0.02 radian. The ZF detection of the MUs' transmit signals $\boldsymbol{w}(k)$ based on the estimated MIMO channel matrix $\widehat{\boldsymbol{H}}$ obtained in a typical identification run is illustrated in Fig. 5. As expected, the scaling of $\boldsymbol{w}(k)$ is the scaling of $\boldsymbol{x}(k)$ 'amplifying' by the HPAs' gains. By passing $\widehat{w}_{m}(k)$ through the estimated Bspline inversion $\left(\widehat{\zeta}_{m} \widehat{\Psi}\right)^{-1}(\cdot)$ to compensate for the nonlinear distortion of the transmitter HPA, for $1 \leq m \leq 3$, the detected MUs' data are obtained, which are shown in Fig. 6. By comparing Fig. 6 with Fig. 2, it can be seen that the correct 64-QAM constellation is restored.

The existing multiuser detection schemes for MIMO are typically based on a linear estimation of the MIMO channel, which can no longer work for nonlinear MIMO uplink. To demonstrate this, we also implement the linear ZF multiuser detection for this MIMO Hammerstein channel. Specifically, we first estimate the 'equivalent' linear MIMO channel matrix $\widehat{\boldsymbol{H}}^{[0]}$ using the LS estimate of (34). This estimated linear MIMO channel matrix $\widehat{\boldsymbol{H}}^{[0]}$ obtained over 100 identification runs is also listed in Table II. Then the linear ZF multiuser detection of $\boldsymbol{x}(k)$ is carried out by

$$
\widehat{\boldsymbol{x}}^{[0]}(k)=\left(\left(\widehat{\boldsymbol{H}}^{[0]}\right)^{\mathrm{H}} \widehat{\boldsymbol{H}}^{[0]}\right)^{-1}\left(\widehat{\boldsymbol{H}}^{[0]}\right)^{\mathrm{H}} \boldsymbol{y}(k) .
$$

The linear ZF detection of $\boldsymbol{x}(k)$ so obtained with $\widehat{\boldsymbol{H}}^{[0]}$ identified at a typical identification run is depicted in Fig. 7. By comparing Fig. 7 with Fig. 2, it is clear that although the scaling of $\boldsymbol{x}(k)$ is correctly recovered, the correct 64QAM constellation is not restored, which is hardly surprising because the nonlinear distortion of the MUs' transmit HPAs is not compensated.

Interestingly, it can be seen that within a complex-value scaling, Fig. 5 and Fig. 7 are very similar. This is because the linear LS estimate $\widehat{\boldsymbol{H}}^{[0]}$ of (34) may be considered as an 'unbiased' estimate of the MIMO channel matrix $\boldsymbol{H}$ scaled by the MUs' transmit HPAs' complex-valued gains, although its estimation accuracy is rather poor. To see this, in Table II, we also divide each column of $\widehat{\boldsymbol{H}}^{[0]}$ by its first-row element, and 


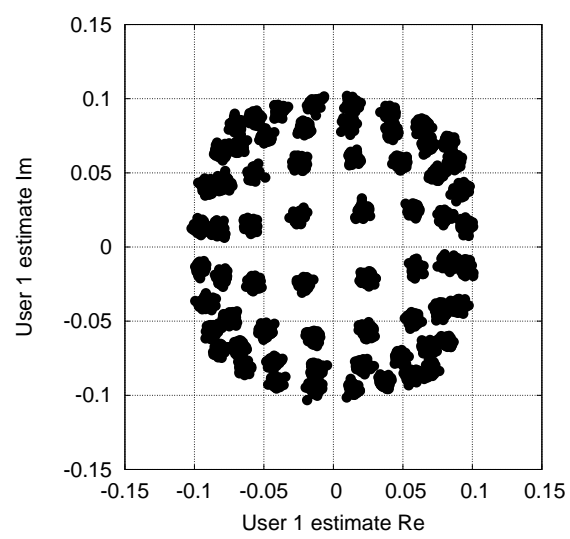

(a)

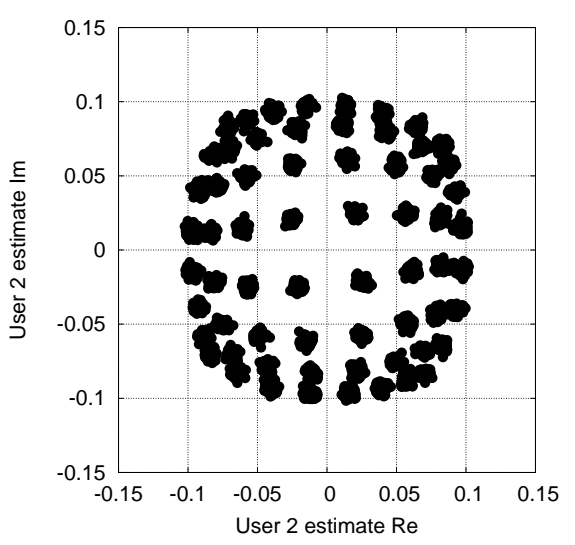

(b)

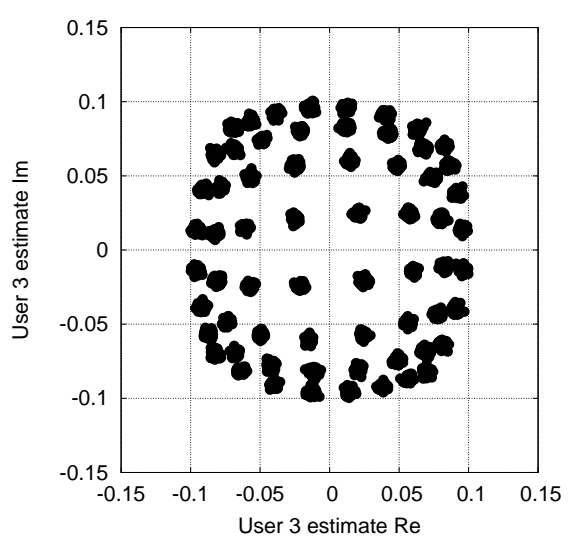

(c)

Fig. 7. Linear ZF detection of $\boldsymbol{x}(k)$ using the estimated linear MIMO channel matrix $\widehat{\boldsymbol{H}}^{[0]}$ obtained by the linear channel estimation scheme at a typical identification run under OBO of $3 \mathrm{~dB}$ and average SNR of $25 \mathrm{~dB}$ : (a) $\widehat{x}_{1}^{[0]}(k)$, (b) $\widehat{x}_{2}^{[0]}(k)$, and (c) $\widehat{x}_{3}^{[0]}(k)$.

the resulting MIMO channel matrix estimate $\left[\widehat{h}_{l, m}^{[0]} / \widehat{h}_{1, m}^{[0]}\right]$ is also shown in Table II. Observe that this scaled or 'normalized' linear LS estimate is an unbiased estimate of the true MIMO channel matrix $\boldsymbol{H}$. Considering that this linear LS estimate is the initial estimate of the MIMO channel matrix in our TL-3S-ALS estimator, it is not surprising that our proposed TL-3S-ALS estimator converges very fast, specifically within $\tau_{\max }=1$ or 2 outer iteration. Hence, our estimation results also provide the empirical evidence to support Remark 3. Moreover, by comparing the scaled linear LS estimate $\widehat{\boldsymbol{H}}^{[0]}$ with our B-spline approach based estimate $\widehat{\boldsymbol{H}}$, it can readily be seen that with only one or two iterations, the estimation accuracy of the latter is significantly better than that of the former. Specifically, the estimation error standard deviations of $\widehat{\boldsymbol{H}}$ are around five times smaller than those of the initial $\widehat{\boldsymbol{H}}^{[0]}$. This clearly demonstrates the estimation efficiency of our proposed TL-3S-ALS estimator.

\section{Bit error rate performance}

Next we randomly generate the MIMO channel matrix $\boldsymbol{H}$ by drawing its entries $h_{l, m}$ for $1 \leq l \leq L$ and $1 \leq m \leq M$ according to the distribution $\mathcal{C N}(0,1)$. Given each true MIMO channel realization $\boldsymbol{H}$, for each given $\mathrm{OBO}$ value and each given average SNR value, we first apply our proposed TL-3SALS algorithm to provide the MIMO channel matrix estimate $\widehat{\boldsymbol{H}}$, the BS estimates $\widehat{\zeta}_{m} \widehat{\Psi}(\cdot)$ of the transmitters' HPAs and their BS inversions $\widehat{\Phi}(\cdot)$ for $1 \leq m \leq 3$ in the training phase. The obtained $\widehat{\boldsymbol{H}}$ and $\widehat{\Phi}(\cdot)$ for $1 \leq m \leq 3$ are used for multiuser detection in the data transmission. For each channel realization, $10^{8}$ 64-QAM data symbol vectors are transmitted, and the bit error rate (BER) of each user is calculated. Obviously, for different channel realizations, the achievable BERs of the three users are different.

The average BER performance over 50 channel realizations obtained by our proposed B-spline based nonlinear multiuser detection scheme and the linear $\mathrm{ZF}$ multiuser detection scheme, respectively, are compared in Fig. 8, given the two OBO values of $3 \mathrm{~dB}$ and $5 \mathrm{~dB}$. Obviously the large the $\mathrm{OBO}$, the less severe the nonlinear distortion of the MUs' transmit HPAs, and hence the better the achievable BER performance. Observe from Fig. 8 that even under the OBO of $5 \mathrm{~dB}$, the classical linear ZF multiuser detection scheme exhibits a high BER floor at the BER level of $10^{-2}$ because it cannot compensate for the nonlinear distortion of the MUs' transmit HPAs. By contrast, our proposed B-spline based nonlinear multiuser detection scheme can effectively compensate for both the linear interference of the MIMO channel and the

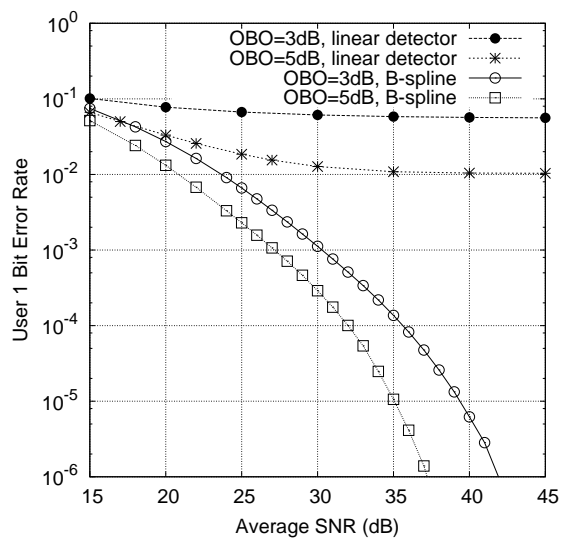

(a)

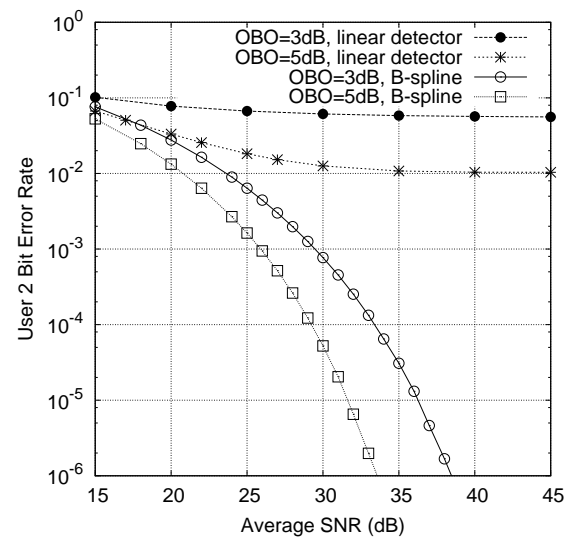

(b)

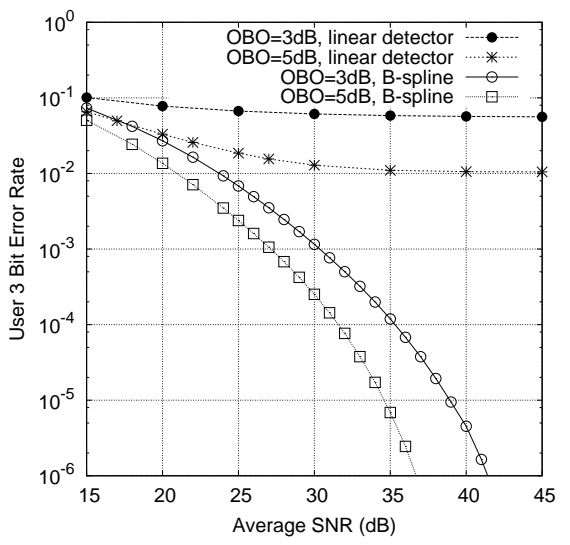

(c)

Fig. 8. Comparison of the average bit error rate performance over 50 channel realizations obtained by the proposed B-spline based nonlinear multiuser detection scheme and the classical linear ZF multiuser detection scheme, respectively, given the two OBO values of $3 \mathrm{~dB}$ and $5 \mathrm{~dB}$ : (a) MU $m=1$, (b) MU $m=2$, and (c) MU $m=3$. 
nonlinear distortion of the MUs' transmit HPAs. Consequently, it dramatically enhances the achievable BER performance, as can be clearly seen from Fig. 8 .

We also repeat all the simulations with $P_{o}=5$ and $N_{R}=$ $N_{I}=10$, and the results obtained are very similar to those reported here for $P_{o}=4$ and $N_{R}=N_{I}=8$.

\section{CONCLUSIONS}

We have proposed a novel nonlinear multiuser detection scheme based on B-spline neural networks for the nonlinear MIMO uplink employing spectral efficient high-order QAM signaling and with nonlinear HPAs at mobile users' transmitters. By adopting a B-spline parameterization of the MUs' nonlinear HPAs, we have derived an efficient TL-3SALS estimator to identify this MIMO Hammerstein channel, including the MIMO channel matrix and the nonlinear mappings of the MUs' nonlinear HPAs. It has been demonstrated that this TL-3S-ALS estimator guarantees to converge very fast to the unbiased estimates of both the MIMO channel matrix and the MUs' nonlinear transmitter mappings with high estimation accuracy. Furthermore, as the direct byproduct of this nonlinear MIMO channel identification, we have also obtained the B-spline inverse mappings of the MUs' nonlinear HPAs. This has allowed us to implement an effective nonlinear multiuser detection scheme by using the ZF detection based on the identified MIMO channel matrix followed by compensating for the nonlinear distortion of the MUs' transmit HPAs with the obtained B-spline inverse mappings. A simulation investigation has been carried out to valid the effectiveness of this B-spline based nonlinear multiuser detection scheme.

This paper is the first to develop a practical and efficient multiuser detection scheme for nonlinear narrowband MIMO systems. Our future work will exploit how to extend the current scheme to space-time equalization of nonlinear frequencyselective MIMO systems.

\section{REFERENCES}

[1] G. J. Foschini, "Layered space-time architecture for wireless communication in a fading environment when using multiple antennas," Bell Labs Tech. J., vol. 1, no. 2, pp. 41-59, 1996.

[2] S. M. Alamouti, "A simple transmit diversity technique for wireless communications," IEEE J. Sel. Areas Commun., vol. 16, no. 8, pp. 14511458., Oct. 1998.

[3] I. E. Telatar, "Capacity of multi-antenna Gaussian channels," European Trans. Commun., vol. 10, no. 2, pp. 585-595, Nov./Dec. 1999.

[4] P. Vandenameele, L. van Der Perre, and M. Engels, Space Division Multiple Access for Wireless Local Area Networks. Boston, MA: Kluwer, 2001.

[5] A. Paulraj, R. Nabar, and D. Gore, Introduction to Space-Time Wireless Communications. Cambridge, U.K.: Cambridge Univ. Press, 2003.

[6] A. J. Paulraj, D. A. Gore, R. U. Nabar, and H. Bolcskei, "An overview of MIMO communications - a key to gigabit wireless," Proc. IEEE, vol. 92, no. 2, pp. 198-218, Feb. 2004.

[7] S. Sugiura, S. Chen, and L. Hanzo, "MIMO-aided near-capacity turbo transceivers: Taxonomy and performance versus complexity," IEEE Commun. Surveys and Tutorials, vol 14, no. 2, pp. 421-442, Secondquarter, 2012.

[8] F. Rusek, et al., "Scaling up MIMO: Opportunities and challenges with very large arrays," IEEE Signal Proces. Mag., vol. 30, no. 1, pp. 40-60, Jan. 2013.

[9] H. Q. Ngo, E. G. Larsson, and T. L. Marzetta, "Energy and spectral efficiency of very large multiuser MIMO systems," IEEE Trans. Commun., vol. 61, no. 4, pp. 1436-1449, Apr. 2013.
[10] E. G. Larsson, O. Edfors, F. Tufvesson, and T. L. Marzetta, "Massive MIMO for next generation wireless systems," IEEE Commun. Mag., vol. 52, no. 2, pp. 186-195, Feb. 2014.

[11] J. Yang and S. Roy, "On joint transmitter and receiver optimization for multiple-input-multiple-output (MIMO) transmission systems," IEEE Trans. Commun., vol. 42, no. 12, pp. 3221-3231, Dec. 1994.

[12] H. Sampath, P. Stoica, and A. Paulraj, "Generalized linear precoder and decoder design for MIMO channels using the weighted MMSE criterion," IEEE Trans. Commun., vol. 49, no. 12, pp. 2198-2206, Dec. 2001.

[13] H. Sampath and A. Paulraj, "Linear precoding for space-time coded systems with known fading correlations," IEEE Commun. Lett., vol. 6, no. 6, pp. 239-241, Jun. 2002.

[14] Y. Jiang, J. Li, and W. W. Hager, "Joint transceiver design for MIMO communications using geometric mean decomposition," IEEE Trans. Signal Process., vol. 53, no. 10, pp. 3791-3803, Oct. 2005.

[15] S. Chen, N. N. Ahmad, and L. Hanzo, "Adaptive minimum bit error rate beamforming," IEEE Trans. Wirel. Commun., vol.4, no. 2, pp. 341-348, Mar. 2005.

[16] S. Chen, L. Hanzo, and A. Livingstone, "MBER Space-time decision feedback equalization assisted multiuser detection for multiple antenna aided SDMA systems," IEEE Trans. Signal Process., vol. 54, no. 8, pp. 3090-3098, Aug. 2006

[17] D. P. Palomar and Y. Jiang, "MIMO transceiver designs via majorization theory," Foundations and Trends in Commun. and Infor. Theory, vol. 3, no. 4-5, pp 331-551, Jun. 2007.

[18] S. Chen, A. Livingstone, H.-Q. Du, and L. Hanzo, "Adaptive minimum symbol error rate beamforming assisted detection for quadrature amplitude modulation," IEEE Trans. Wirel. Commun., vol. 7, no. 4, pp. 11401145, Apr. 2008.

[19] S. Chen, W. Yao, and L. Hanzo, "Semi-blind adaptive spatial equalisation for MIMO systems with high-order QAM signalling," IEEE Trans. Wirel. Commun., vol. 7, no. 11, pp. 4486-4491, Nov. 2008.

[20] S. Chen and L. Hanzo, "Fast converging semi-blind space-time equalisation for dispersive QAM MIMO systems," IEEE Trans. Wirel. Commun., vol. 8, no. 8, pp. 3969-3974, Aug. 2009.

[21] W. Yao, S. Chen, S. Tan, and L. Hanzo, "Minimum bit error rate multiuser transmission designs using particle swarm optimisation," IEEE Trans. Wirel. Commun., vol. 8, no. 10, pp. 5012-5017, Oct. 2009.

[22] W. Yao, S. Chen, and L. Hanzo, "A transceiver design based on uniform channel decomposition and MBER vector perturbation," IEEE Trans. Veh. Techno., vol. 59, no. 6, pp. 3153-3159, Jul. 2010.

[23] W. Yao, S. Chen, and L. Hanzo, "Generalised MBER-based vector precoding design for multiuser transmission," IEEE Trans. Veh. Techno., vol. 60, no. 2, pp. 739-745, Feb. 2011.

[24] S. Sugiura, S. Chen, and L. Hanzo, "Generalized space-time shift keying designed for flexible diversity-, multiplexing- and complexity-tradeoffs," IEEE Trans. Wirel. Commun., vol. 10, no. 4, pp. 1144-1153, Apr. 2011.

[25] P. Zhang, S. Chen, and L. Hanzo, "Two-tier channel estimation aided near-capcity MIMO transceivers relaying on norm-based joint transmit and receive antenna selection," IEEE Trans. Wirel. Commun., vol. 14, no. 1, pp. 122-137, Jan. 2015.

[26] L. Hanzo, S. X. Ng, T. Keller, and W. Webb, Quadrature Amplitude Modulation: From Basics to Adaptive Trellis-Coded, Turbo-Equalised and Space-Time Coded OFDM, CDMA and MC-CDMA Systems. Chichester, UK: John Wiley, 2004.

[27] A. A. M. Saleh, "Frequency-independent and frequency-dependent nonlinear models of TWT amplifiers," IEEE Trans. Commun., vol. COM-29, no. 11, pp. 17151720, Nov. 1981.

[28] M. Honkanen and S.-G. Häggman, "New aspects on nonlinear power amplifier modeling in radio communication system simulations," in Proc. PIMRC'97 (Helsinki, Finland), Sep. 1-4, 1997, pp. 844-848.

[29] C. J. Clark, et al., "Time-domain envelope measurement technique with application to wideband power amplifier modeling," IEEE Trans. Microw. Theory and Tech., vol. 46, no. 12, pp. 2531-2540, Dec. 1998.

[30] C.-S. Choi, et al., "RF impairment models $60 \mathrm{GHz}$ band SYS/PHY simulation," Document IEEE 802.15-06-0477-01-003c, Nov. 2006. https://mentor. ieee.org/802.15/dcn/06/15-06-0477-01-003c-rf -impairment-models-60ghz-band-sysphy -simulation.pdf

[31] V. Erceg, et al., "60 GHz impairments modeling," Document IEEE 802.11-09/1213r1, Nov. 2009.

[32] L. Ding, et al., "A robust digital baseband predistorter constructed using memory polynomials," IEEE Trans. Commun., vol. 52, no. 1, pp. 159165, Jan. 2004. 
[33] D. Zhou and V. E. DeBrunner, "Novel adaptive nonlinear predistorters based on the direct learning algorithm," IEEE Trans. Signal Process., vol. 55, no. 1, pp. 120-133, Jan. 2007

[34] M.-C. Chiu, C.-H. Zeng, and M.-C. Liu, "Predistorter based on frequency domain estimation for compensation of nonlinear distortion in OFDM systems," IEEE Trans. Veh. Techno., vol. 57, no. 2, pp. 882-892, Mar. 2008.

[35] S. Choi, E.-R. Jeong, and Y. H. Lee, "Adaptive predistortion with direct learning based on piecewise linear approximation of amplifier nonlinearity," IEEE J. Sel. Topics Signal Process., vol. 3, no. 3, pp. 397404, Jun. 2009.

[36] V. P. G. Jiménez, Y. Jabrane, A. G. Armada, and B. Ait Es Said, "High power amplifier pre-distorter based on neural-fuzzy systems for OFDM signals," IEEE Trans. Broadcasting, vol.57, no. 1, pp. 149-158, Mar. 2011.

[37] S. Chen, "An efficient predistorter design for compensating nonlinear memory high power amplifier," IEEE Trans. Broadcasting, vol. 57, no. 4, pp. 856-865, Dec. 2011.

[38] S. Chen, X. Hong, Y. Gong, and C. J. Harris, "Digital predistorter design using B-spline neural network and inverse of De Boor algorithm," IEEE Trans. Circuits and Systems I, vol. 60, no. 6, pp. 1584-1594, Jun. 2013.

[39] C. A. R. Fernandes, Nonlinear MIMO Communication Systems: Channel Estimation and Information Recovery Using Volterra Models. $\mathrm{PhD}$ These, Université de Nice Sophia Antipolis, France, 2009.

[40] C. A. R. Fernandes, J. C. M. Mota, and G. Favier, "MIMO Volterra modeling for nonlinear communication channels," J. Brazilian Society Neural Networks, vol. 8, no. 2, pp. 71-92, 2010

[41] C. A. R. Fernandes, G. Favier, and J. C. M. Mota, "PARAFACbased channel estimation and data recovery in nonlinear MIMO spread spectrum communication systems," Signal Process., vol. 91, no. 2, pp. 311-322, Feb. 2011.

[42] C. De Boor, A Practical Guide to Splines. New York: Spring Verlag, 1978.

[43] C. J. Harris, X. Hong, and Q. Gan, Adaptive Modelling, Estimation and Fusion from Data: A Neurofuzzy Approach. Berlin: Springer-Verlag, 2002.

[44] X. Hong and S. Chen, "Modeling of complex-valued Wiener systems using B-spline neural network," IEEE Trans. Neural Networks, vol 22, no. 5, pp. 818-825, May 2011.

[45] S. Chen, X. Hong, J. B. Gao, and C. J. Harris, "Complex-valued B-spline neural networks for modeling and inverting Hammerstein systems," IEEE Trans. Neural Networks and Learning Systems, vol. 25, no. 9, pp. 1673-1685, Sep. 2014.

[46] X. Hong, S. Chen, C. J. Harris, and E. Khalaf, "Single-carrier frequency domain equalization for Hammerstein communication systems using complex-valued neural networks," IEEE Trans. Signal Process., vol. 62, no. 17 , pp. 4467-4478, Sep. 2014.

[47] X. Hong, S. Chen, Y. Gong, and C. J. Harris, "Nonlinear equalization of Hammerstein OFDM systems," IEEE Trans. Signal Process., vol. 62, no 21 , pp. 5629-5639, Nov. 2014.

[48] S. Chen, et al., "Adaptive B-spline neural network based nonlinear equalization for high-order QAM systems with nonlinear transmit high power amplifier,' Digital Signal Process., vol. 40, pp. 238-249, May 2015.

[49] S. Chen, et al., "Single-carrier frequency-domain equalization with hybrid decision feedback equalizer for Hammerstein channels containing nonlinear transmit amplifier," IEEE Trans. Wirel. Commun., vol. 16, no. 5, pp. 3341-3354, May 2017.

[50] S. Chen, et al., "Comparative performance of complex-valued B-spline and polynomial models applied to iterative frequency-domain decision feedback equalization of Hammerstein channels," IEEE Trans. Neural Networks and Learning Systems, vol. 28, no. 12, pp. 2872-2884, Sep. 2017.

[51] J. M. Pena, "B-spline and optimal stability," Mathematics of Computation, vol. 66, no. 220, pp. 1555-1560, Oct. 1997.

[52] T. Lyche and J. M. Pena, "Optimally stable multivariate bases," Advances in Computational Mathematics, vol. 20, nos. 1-3, pp. 149-159, Jan. 2004.

[53] E. Mainar and J. M. Pena, "Optimal stability of bivariate tensor product B-bases," J. Numerical Analysis, Industrial and Applied Mathematics, vol. 6 nos. 3-4, pp. 95-104, 2011.

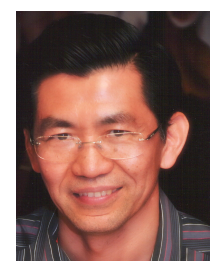

Sheng Chen (M'90-SM'97-F'08) received his BEng degree from the East China Petroleum Institute, Dongying, China, in 1982, and his $\mathrm{PhD}$ degree from the City University, London, in 1986, both in control engineering. In 2005, he was awarded the higher doctoral degree, Doctor of Sciences (DSc), from the University of Southampton, Southampton, UK.

From 1986 to 1999 , He held research and academic appointments at the Universities of Sheffield, Edinburgh and Portsmouth, all in UK. Since 1999, he has been with the School of Electronics and Computer Science, the University of Southampton, UK, where he holds the post of Professor in Intelligent Systems and Signal Processing. Dr Chen's research interests include neural network and machine learning, wireless communications, and adaptive signal processing. He has published over 650 research papers. Professor Chen has 13,800+ Web of Science citations and 28,300+ Google Scholar citations.

Dr. Chen is a Fellow of the United Kingdom Royal Academy of Engineering, a Fellow of IET, a Distinguished Adjunct Professor at King Abdulaziz University, Jeddah, Saudi Arabia, and an original ISI highly cited researcher in engineering (March 2004)

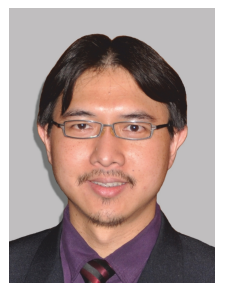

Soon Xin Ng (S'99-M'03-SM'08) received the B.Eng. degree (First class) in electronic engineering and the Ph.D. degree in telecommunications from the University of Southampton, Southampton, U.K., in 1999 and 2002, respectively.

From 2003 to 2006 , he was a postdoctoral research fellow working on collaborative European research projects known as SCOUT, NEWCOM and PHOENIX. Since August 2006, he has been a member of academic staff in the School of Electronics and Computer Science, University of Southampton. He was involved in the OPTIMIX and CONCERTO European projects as well as the IU-ATC and UC4G projects. He was the principal investigator of an EPSRC project-Cooperative Classical and Quantum Communications Systems. He is currently an Associate Professor in telecommunications at the University of Southampton.

His research interests include adaptive coded modulation, coded modulation, channel coding, space-time coding, joint source and channel coding, iterative detection, OFDM, MIMO, cooperative communications, distributed coding, quantum communications, quantum error correction codes, joint wireless-and-optical-fibre communications, game theory, artificial intelligence and machine learning. He has published over 250 papers and co-authored two John Wiley/IEEE Press books in this field.

$\mathrm{He}$ is a Fellow of the Higher Education Academy in the UK, a Chartered Engineer and a Fellow of the IET. He acted as TPC/track/workshop chairs for various conferences. He serves as an associate editor to the IEEE Access and as the editor of Quantum Engineering. He was/is one of the guest editors for the special issues in: IEEE Journal on Selected Areas in Communications (2019) and IEEE Access (2017). He was also one of the editors in KSII Transactions on Internet and Information Systems. He is one of the Founders and Officers of the IEEE Quantum Communications \& Information Technology Emerging Technical Subcommittee (QCIT-ETC).

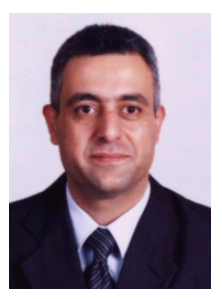

Emad F. Khalaf received his B.Eng. and M.Eng. degrees in Computer Engineering from Wroclaw University of Technology in Poland, in 1992, as one certificate, and the Ph.D. degree in Computer networks from Wroclaw University of Technology, in Poland, in 2002.

From 2003 to 2011, he was an assistant Professor at Computer Engineering Department, Faculty of Engineering, Philadelphia University. Since 2012, he is an assistant Professor at Electrical and Computer Engineering Department, Faculty of Engineering, King Abdulaziz University, Jeddah, Saudi Arabia. His research interests are in network security, speech classification and recognition, image and signal processing. 


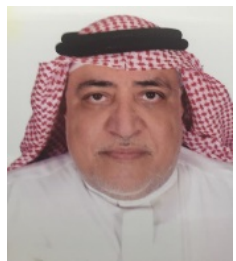

Ali Morfeq received the M.Sc. degree from Oregon State University, Corvallis, OR, USA, in 1985, and the Ph.D. degree from University of Colorado at Boulder, Boulder, CO, USA, in 1990.

$\mathrm{He}$ is the vice dean of faculty of Engineering at King Abdulaziz University (KAU) since 2013. $\mathrm{He}$ is an associate professor there. He also served at $\mathrm{KAU}$ in several administration positions, and as the Head of many academic and administrative committees. Additionally, he worked as a Consultant to many firms including big industrial companies and several hospitals. His work involved automation of office environments and the supporting databases. He had, and still has, many graduate students working in different areas such decision support systems, data marts, and data warehouses.

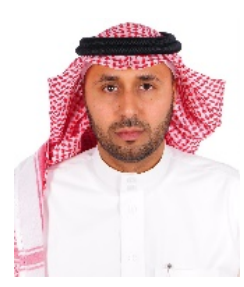

Naif D. Alotaibi completed his B.Sc. in Computer Engineering from KAU in Jeddah, Saudi Arabia, in 2000. He received his Master and $\mathrm{PhD}$ degrees in Information Technology from Queensland University of Technology (QUT) in Brisbane, Australia, in 2006 and 2012, respectively.

In 2013, he joined the Electrical and Computer Engineering Department at the Faculty of Engineering, King Abdulaziz University, Jeddah, Saudi Arabia as an assistant professor. His research interests include IT infrastructure, computer network quality and network QoS, and Quality Measurement. 